Article

\title{
Narrow Genetic Diversity of Wolbachia Symbionts in Acrididae Grasshopper Hosts (Insecta, Orthoptera)
}

\author{
Yury Ilinsky ${ }^{1,2, *}$, Mary Demenkova ${ }^{1}$, Roman Bykov ${ }^{1}$ (I) and Alexander Bugrov ${ }^{3,4}$ \\ 1 Laboratory of Molecular Genetics of Insects, Institute of Cytology and Genetics SB RAS, \\ 630090 Novosibirsk, Russia; judina@bionet.nsc.ru (M.D.); bykovra@bionet.nsc.ru (R.B.) \\ 2 Center for Mitochondrial Functional Genomics, Immanuel Kant Baltic Federal University, \\ 236041 Kaliningrad, Russia \\ 3 Faculty of Natural Sciences, Novosibirsk State University, 630090 Novosibirsk, Russia; bugrov04@yahoo.co.uk \\ 4 Institute of Systematics and Ecology of Animals SB RAS, 630091 Novosibirsk, Russia \\ * Correspondence: paulee@bionet.nsc.ru
}

Citation: Ilinsky, Y.; Demenkova, M.; Bykov, R.; Bugrov, A. Narrow Genetic Diversity of Wolbachia Symbionts in Acrididae Grasshopper Hosts (Insecta, Orthoptera). Int. J. Mol. Sci. 2022, 23, 853. https://doi.org/ $10.3390 /$ ijms 23020853

Academic Editor: Sergei A Subbotin

Received: 14 December 2021

Accepted: 11 January 2022

Published: 13 January 2022

Publisher's Note: MDPI stays neutral with regard to jurisdictional claims in published maps and institutional affiliations.

Copyright: (C) 2022 by the authors. Licensee MDPI, Basel, Switzerland. This article is an open access article distributed under the terms and conditions of the Creative Commons Attribution (CC BY) license (https:// creativecommons.org/licenses/by/ $4.0 /)$.

\begin{abstract}
Bacteria of the Wolbachia genus are maternally inherited symbionts of Nematoda and numerous Arthropoda hosts. There are approximately 20 lineages of Wolbachia, which are called supergroups, and they are designated alphabetically. Wolbachia strains of the supergroups A and B are predominant in arthropods, especially in insects, and supergroup F seems to rank third. Host taxa have been studied very unevenly for Wolbachia symbionts, and here, we turn to one of largely unexplored insect families: Acrididae. On the basis of five genes subject to multilocus sequence typing, we investigated the incidence and genetic diversity of Wolbachia in 41 species belonging three subfamilies (Gomphocerinae, Oedipodinae, and Podisminae) collected in Turkey, Kazakhstan, Tajikistan, Russia, and Japan, making 501 specimens in total. Our results revealed a high incidence and very narrow genetic diversity of Wolbachia. Although only the strains belonging to supergroups A and B are commonly present in present, the Acrididae hosts here proved to be infected with supergroups B and F without A-supergroup variants. The only trace of an A-supergroup lineage was noted in one case of an inter-supergroup recombinant haplotype, where the ftsZ gene came from supergroup A, and the others from supergroup B. Variation in the Wolbachia haplotypes in Acrididae hosts within supergroups B and F was extremely low. A comprehensive genetic analysis of Wolbachia diversity confirmed specific features of the Wolbachia allelic set in Acrididae hosts. This result can help to elucidate the crucial issue of Wolbachia biology: the route(s) and mechanism(s) of Wolbachia horizontal transmission.
\end{abstract}

Keywords: Acrididae; horizontal transmission; multilocus sequence typing; recombination; population; symbiont; Wolbachia

\section{Introduction}

Bacteria of the Wolbachia genus are maternally inherited symbionts of Nematoda and numerous Arthropoda hosts. The wide distribution of Wolbachia in insect hosts resembles a pandemic [1]. To spread through a host population, the Wolbachia symbionts induce reproductive abnormalities such as cytoplasmic incompatibility, parthenogenesis, male killing, and feminisation [2]. Additionally, there is evidence of mutualistic effects between Wolbachia symbionts and their hosts [3]. There are 20 lineages of Wolbachia called supergroups, and these are designated alphabetically with some omissions [4-6]. Strains belonging to supergroups $\mathrm{A}$ and $\mathrm{B}$ are predominant in arthropods, especially in insects, and the F supergroup seems to take third place and is partitularly detectable in Nematoda [7], Coleoptera [8-10], Diptera [11], Hemiptera [12], Hymenoptera [1,13,14], Isopoda [15], Odonata [16], Scorpiones [17], Strepsiptera [18], and Termites [1,19-21]. Strains of other Wolbachia lineages are found much less frequently and are often associated with a specific 
host taxon. Similar or even the same Wolbachia strains can be found in unrelated hosts, implying the horizontal transmission of the symbiont. The routes of interspecies transmission are not well understood at present. Predation, host-parasitoid interactions, and feeding on a common substrate are possible mechanisms [22-31]; however, the main transmission pathway remains unknown.

Acrididae grasshoppers comprise more than 10,000 species that are widespread in nearly all terrestrial landscapes. Due to the great importance of Acrididae grasshoppers as agricultural pests, much research attention has been given to this taxon.

Data on Wolbachia in Acrididae are fragmentary. Podisma pedestris [32] and Podisma sapporensis [33] are known to be affected by Wolbachia infection. Samples of Acrida willemsei, Calliptamus italicus, Ceracris fasciata, Catantops humilis, Chorthippus brunneus, Hieroglyphus banian, Melanoplus sp., Oxya ntricata, and Oxya japonica have been reported to be uninfected [23,34-37]. A series of studies have been performed on populations of Chorthippus parallelus inhabiting French and Spanish territories [38-45], where investigators have reported Wolbachia strains of supergroups B and $\mathrm{F}$ and even $\mathrm{B} / \mathrm{F}$ recombinants.

Here, we evaluated the genetic diversity of Wolbachia in members of the Acrididae family. In particular, we screened specimens from three subfamilies (Gomphocerinae, Oedipodinae, and Podisminae) collected in Turkey, Kazakhstan, Tajikistan, Russia, and Japan and characterised Wolbachia isolates by a multilocus sequence typing (MLST) protocol that included five housekeeping genes [12]. We aimed to investigate Wolbachia incidence in Acrididae hosts and to determine whether there is a Wolbachia genetic pattern that is specific to Acrididae.

\section{Results}

\subsection{Wolbachia Occurrence in Acrididae}

We examined the infection status in 41 Acrididae species and found 28 Wolbachia-infected species (Table 1). Only two species have been analysed previously: P. sapporensis [33] and P. pedestris [32]. The symbionts were found in all of the subfamilies under study: Gomphocerinae, Oedipodinae, and Podisminae. There were no noticeable differences in Wolbachia prevalence between the regions and the between specimen collection years. The estimation of infection prevalence was not the aim of our study because we mainly tested somatic tissues that may only reflect the lowest rate boundary. Nevertheless, we registered high Wolbachia prevalence in Ch. biguttulus (174/198), Ch. fallax (16/17), and P. montanus (40/50). High Wolbachia prevalence in P. sapporensis populations was determined based on gonad tissues and was reported earlier [33]; here, we screened an additional 18 specimens for infection and confirmed the previous conclusion. Sample sizes were not sufficient to make firm conclusions about other species.

\subsection{Genetic Diversity of Wolbachia Isolates}

A total of 44 Wolbachia isolates were characterised by the MLST protocol [12]; 43 were unique according to the species-site-year combination (Table 2). We managed to amplify all five loci for 39 isolates, and for 35 isolates, we obtained unambiguous allele sequences. For many isolates, there were difficulties in obtaining complete MLST profiles. In two cases (ftsZ of i-1 and gatB of i-15), we failed to obtain amplicons, even when using nested PCR. For several isolates, we could not obtain good quality sequences; there were double chromatogram peaks for certain positions or loci, or in some cases, even repeated double peaks in the bulk of a sequence. 
Table 1. The Acrididae specimens and Wolbachia infection.

\begin{tabular}{|c|c|c|c|}
\hline Subfamily & Species & $\begin{array}{l}\text { Region and Year of } \\
\text { Collection }\end{array}$ & $\begin{array}{l}\text { No. of Infected } \\
\text { Specimens/Total }\end{array}$ \\
\hline \multirow[t]{29}{*}{ Gomphocerinae } & Arcyptera (Arcyptera) fusca (Pallas, 1773) & Russia, Altai Mts, 2017 & $2 / 2$ \\
\hline & $\begin{array}{c}\text { Arcyptera (Pararcyptera) microptera (Fischer von } \\
\text { Waldheim, 1833) }\end{array}$ & Russia, Altai Mts, 2017 & $0 / 5$ \\
\hline & Chorthippus (Chorthippus) albomarginatus (De Geer, 1773) & $\begin{array}{l}\text { Russia, Irkutsk region, } \\
2016\end{array}$ & $10 / 10$ \\
\hline & Chorthippus (Glyptobothrus) apricarius (Linnaeus, 1758) & $\begin{array}{l}\text { Russia, Irkutsk region, } \\
2016\end{array}$ & $7 / 7$ \\
\hline & Chorthippus (Glyptobothrus) biguttulus (Linnaeus, 1758) & East Kazakhstan, 2007 & $0 / 6$ \\
\hline & $-"$ & $\begin{array}{l}\text { Russia, Novosibirsk } \\
\text { region, } 2017\end{array}$ & $174 / 198$ \\
\hline & $-{ }^{\prime \prime}-$ & Russia, Irkutsk region & $4 / 6$ \\
\hline & $-{ }^{\prime \prime}-$ & Russia, Altai Mts, 2015 & $5 / 5$ \\
\hline & $\begin{array}{c}\text { Chorthippus (Altichorthippus) intermedius } \\
\text { (Bey-Bienko, 1926) }\end{array}$ & Russia, Altai Mts, 2003 & $0 / 8$ \\
\hline & Chorthippus (Glyptobothrus) mollis (Charpentier, 1825) & Russia, Altai Mts, 2003 & $2 / 5$ \\
\hline & $-{ }^{\prime \prime}-$ & Turkey, 2003 & $0 / 9$ \\
\hline & Chorthippus fallax (Zubovski, 1900) & $\begin{array}{l}\text { Russia, Novosibirsk } \\
\text { region, } 2017\end{array}$ & $16 / 17$ \\
\hline & Chorthippus hammarstroemi (Miram, 1907) & Russia, Altai Mts, 2003 & $0 / 5$ \\
\hline & Dociostaurus (Kazakia) brevicollis (Eversmann, 1848) & East Kazakhstan, 2007 & $3 / 7$ \\
\hline & Dociostaurus (Kazakia) tartarus (Stshelkanovtzev, 1921) & East Kazakhstan, 2007 & $0 / 5$ \\
\hline & Eclipophleps glacialis (Bey-Bienko, 1933) & Russia, Altai Mts, 2003 & $2 / 8$ \\
\hline & Eremippus simplex (Eversmann, 1859) & East Kazakhstan, 2007 & $0 / 2$ \\
\hline & Euthystira brachyptera (Ocskay, 1826) & Russia, Altai Mts, 2003 & $0 / 2$ \\
\hline & Gomphocerippus rufus (Linnaeus, 1758) & $\begin{array}{l}\text { Russia, Novosibirsk } \\
\text { region, } 2017\end{array}$ & $1 / 7$ \\
\hline & Megaulacobothrus aethalinus (Zubovski, 1899) & Russia, Altai Mts, 2003 & $0 / 6$ \\
\hline & Omocestus (Omocestus) viridulus (Linnaeus, 1758) & $\begin{array}{l}\text { Russia, Novosibirsk } \\
\text { region, } 2009\end{array}$ & $3 / 4$ \\
\hline & $-"$ & Russia, Altai Mts, 2017 & $1 / 1$ \\
\hline & $\begin{array}{c}\text { Omocestus (Omocestus) haemorrhoidalis } \\
\text { (Charpentier, 1825) }\end{array}$ & Russia, Altai Mts, 2017 & $1 / 2$ \\
\hline & Podismopsis altaica (Zubovski, 1900) & Russia, Altai Mts, 2003 & $3 / 4$ \\
\hline & Podismopsis genicularibus (Shiraki, 1910) & Russia, Sakhalin Is., 2010 & $2 / 3$ \\
\hline & Pseudochorthippus montanus (Charpentier, 1825) & $\begin{array}{l}\text { Russia, Novosibirsk } \\
\text { region, } 2017\end{array}$ & $40 / 50$ \\
\hline & Pseudochorthippus parallelus (Zetterstedt, 1821) & $\begin{array}{l}\text { Russia, Novosibirsk } \\
\text { region, } 2017\end{array}$ & $5 / 5$ \\
\hline & Stauroderus scalaris (Fischer von Waldheim, 1846) & Russia, Altai Mts, 2017 & $8 / 8$ \\
\hline & Stenobothrus eurasius (Zubovski, 1898) & Russia, Altai Mts, 2003 & $0 / 2$ \\
\hline \multirow[t]{3}{*}{ Oedipodinae } & Bryodema gebleri (Fischer von Waldheim, 1836) & Russia, Altai Mts, 2003 & $2 / 3$ \\
\hline & $-" \prime$ & Russia, Altai Mts, 2017 & $2 / 2$ \\
\hline & Bryodema tuberculata (Fabricius, 1775) & Russia, Altai Mts, 2003 & $0 / 3$ \\
\hline
\end{tabular}


Table 1. Cont.

\begin{tabular}{|c|c|c|c|}
\hline Subfamily & Species & $\begin{array}{l}\text { Region and Year of } \\
\text { Collection }\end{array}$ & $\begin{array}{l}\text { No. of Infected } \\
\text { Specimens/Total }\end{array}$ \\
\hline & Locusta migratoria (Linnaeus, 1758) & Central Kazakhstan, 2007 & $0 / 4$ \\
\hline & Oedaleus decorus (Germar, 1825) & Russia, Altai Mts, 2017 & $1 / 1$ \\
\hline & $-{ }^{\prime \prime}-$ & East Kazakhstan, 2007 & $3 / 4$ \\
\hline & $-"-$ & Tadzhikistan, 2009 & $1 / 1$ \\
\hline & Psophus stridulus (Linnaeus, 1758) & Russia, Altai Mts, 2003 & $0 / 1$ \\
\hline & Pyrgodera armata (Fischer von Waldheim, 1846) & East Kazakhstan, 2007 & $0 / 4$ \\
\hline \multirow[t]{19}{*}{ Podisminae } & Anapodisma miramae (Dovnar-Zapolskij, 1932) & $\begin{array}{l}\text { Russia, Maritima region of } \\
\text { Far East, } 2008\end{array}$ & $1 / 1$ \\
\hline & Bohemanella frigida (Boheman, 1846) & Russia, Altai Mts, 2003 & $1 / 3$ \\
\hline & Ognevia longipennis (Shiraki, 1910) & Japan, Hokkaido, 2005 & $4 / 4$ \\
\hline & $-{ }^{\prime \prime}-$ & $\begin{array}{c}\text { Russia, Altai } \\
\text { Mts, Edigan, } 2003\end{array}$ & $5 / 5$ \\
\hline & Podisma pedestris (Linnaeus, 1758) & Russia, Altai Mts, 2003 & $5 / 5$ \\
\hline & $-{ }^{\prime \prime}-$ & Russia, Altai Mts, 2016 & $1 / 4$ \\
\hline & Podisma kanoi (Storozhenko, 1994) & Japan, Honshu, 2005 & $1 / 1$ \\
\hline & Podisma sapporensis (Shiraki, 1910) & $\begin{array}{l}\text { Japan, Hokkaido Is, Tanno } \\
\text { town vicinities, } 2005\end{array}$ & $5 / 5$ \\
\hline & $-{ }^{\prime \prime}-$ & $\begin{array}{l}\text { Japan, Hokkaido Akan } \\
\text { town vicinities, } 2005\end{array}$ & $5 / 5$ \\
\hline & $-{ }^{\prime \prime}-$ & $\begin{array}{c}\text { Japan, Hokkaido, Yotei } \\
\text { Mt., } 2005\end{array}$ & $5 / 5$ \\
\hline & $-{ }^{\prime \prime}-$ & $\begin{array}{l}\text { Japan, Hokkaido, } \\
\text { Disengen Mt., } 2005\end{array}$ & $5 / 5$ \\
\hline & $-"-$ & $\begin{array}{c}\text { Japan, Hokkaido, } \\
\text { Naganuma town vicinities, } \\
2005\end{array}$ & $5 / 5$ \\
\hline & - & $\begin{array}{c}\text { Japan, Hokkaido, Teine } \\
\text { Mt., } 2005\end{array}$ & $10 / 10$ \\
\hline & $-{ }^{\prime \prime}-$ & $\begin{array}{c}\text { Japan, Japan, Hokkaido, } \\
\text { Shimokawa town } \\
\text { vicinities, } 2005\end{array}$ & $3 / 3$ \\
\hline & Podisma tyatiensis (Bugrov \& Sergeev, 1997) & $\begin{array}{l}\text { Russia, Kuril Arch., } \\
\text { Kunashir Is, } 2001\end{array}$ & $1 / 1$ \\
\hline & Prumna littoralis (Tarbinsky, 1932) & $\begin{array}{l}\text { Russia, Maritima region of } \\
\text { Far East, } 2008\end{array}$ & $1 / 1$ \\
\hline & Prumna primnoa (Motschulsky, 1846) & Russia, Sakhalin Is, 2010 & $5 / 5$ \\
\hline & Prumna ussuriensis (Tarbinsky, 1930) & $\begin{array}{l}\text { Russia, Maritima region of } \\
\text { Far East, } 2008\end{array}$ & $1 / 5$ \\
\hline & Sinopodisma punctata (Mishchenko, 1954) & $\begin{array}{l}\text { Japan, Ryukyu Arch., } \\
\text { Ishigaki Is, } 2005\end{array}$ & $0 / 3$ \\
\hline
\end{tabular}


Table 2. Wolbachia MLST profiles of the analysed Acrididae isolates.

\begin{tabular}{|c|c|c|c|c|c|c|c|c|}
\hline Isolate & Species (Region, Year) & Supergroup & gatB & $\operatorname{cox} \mathbf{A}$ & hсpA & ftsZ & fbpA & $\begin{array}{l}\text { Sequ-Ence } \\
\text { Type * }\end{array}$ \\
\hline $\mathrm{i}-1$ & Arcyptera fusca (Altai, 2017) & $\mathrm{F}$ & $\begin{array}{c}\sim 73 * * \\
(\mathrm{MZ} 816480)\end{array}$ & $\begin{array}{c}\sim 63 \\
(\mathrm{MZ} 816523)\end{array}$ & $\begin{array}{c}\sim 261 \\
(\mathrm{MZ} 816567)\end{array}$ & No $* * *$ & $\begin{array}{c}410 \\
(\mathrm{MZ} 816669)\end{array}$ & not full \\
\hline $\mathrm{i}-2$ & $\begin{array}{l}\text { Chorthippus albomarginatus } \\
\text { (Irkutsk, 2016) }\end{array}$ & B & $\begin{array}{c}134 \\
(\mathrm{MZ} 816445)\end{array}$ & $\begin{array}{c}168 \\
(\mathrm{MZ} 816488)\end{array}$ & $\begin{array}{c}\sim 6 \\
(\mathrm{MZ} 816532)\end{array}$ & $\begin{array}{c}106 \\
(\mathrm{MZ} 816581)\end{array}$ & $\begin{array}{c}197 \\
(\mathrm{MZ816634)}\end{array}$ & $h^{S T}-1$ \\
\hline i-3 & $\begin{array}{l}\text { Ch. Apricarius } \\
\text { (Irkutsk, 2016) }\end{array}$ & B & $\begin{array}{c}134 \\
(\mathrm{MZ} 816446)\end{array}$ & $\begin{array}{c}14 \\
(\mathrm{MZ} 816489)\end{array}$ & $\begin{array}{c}\sim 6 \\
(\mathrm{MZ} 816533)\end{array}$ & $\begin{array}{c}106 \\
(\mathrm{MZ816582})\end{array}$ & $\begin{array}{c}197 \\
(\mathrm{MZ816635)}\end{array}$ & $h^{\mathrm{ST}}-2$ \\
\hline $\mathrm{i}-4$ & $\begin{array}{l}\text { Ch. Biguttulus } \\
\text { (Irkutsk, 2016) }\end{array}$ & B & 9 (MZ816447) & $\begin{array}{c}133 \\
(\mathrm{MZ} 816490)\end{array}$ & $\begin{array}{c}\sim 6 \\
(\mathrm{MZ} 816534)\end{array}$ & $\begin{array}{c}106 \\
(\mathrm{MZ} 816583)\end{array}$ & $\begin{array}{c}197 \\
(\mathrm{MZ} 816636)\end{array}$ & $h^{S T}-3$ \\
\hline i-5 & $\begin{array}{c}\text { Ch. Bigut- } \\
\text { tulus (Novosibirsk, 2017) }\end{array}$ & B & 9 (MZ816448) & $\begin{array}{c}133 \\
(\mathrm{MZ} 816491)\end{array}$ & $\begin{array}{c}6 \\
(\mathrm{MZ} 816535)\end{array}$ & $\begin{array}{c}106 \\
(\mathrm{MZ} 816584)\end{array}$ & $\begin{array}{c}197 \\
(\mathrm{MZ816637)}\end{array}$ & $\mathrm{h}^{\mathrm{ST}}-4$ \\
\hline i-6 & Ch. Biguttulus (Altai, 2015) & B & 9 (MZ816481) & $\begin{array}{c}133 \\
(\mathrm{MZ} 816524)\end{array}$ & $?^{* * * *}$ & $?$ & $?$ & not full \\
\hline $\mathrm{i}-7$ & Ch. Mollis (Altai, 2003) & B & 9 (MZ816449) & $\begin{array}{c}133 \\
(\mathrm{MZ} 816492)\end{array}$ & $\begin{array}{c}6 \\
(\mathrm{MZ} 816536) \\
\end{array}$ & $\begin{array}{c}106 \\
(\mathrm{MZ} 816585)\end{array}$ & $\begin{array}{c}197 \\
(\mathrm{MZ} 816638)\end{array}$ & $\mathrm{h}^{\mathrm{ST}}-4$ \\
\hline i-8 & $\begin{array}{c}\text { Ch. Fallax } \\
\text { (Novosibirsk, 2017) }\end{array}$ & B & 9 (MZ816450) & $\begin{array}{c}133 \\
(\mathrm{MZ} 816493)\end{array}$ & $\begin{array}{c}6 \\
(\mathrm{MZ} 816537)\end{array}$ & $\begin{array}{c}106 \\
(\mathrm{MZ} 816586)\end{array}$ & $\begin{array}{c}197 \\
(\mathrm{MZ816639)}\end{array}$ & $h^{\mathrm{ST}}-4$ \\
\hline i-9 & $\begin{array}{l}\text { Dociostaurus brevicollis } \\
\text { (Kazakhstan, 2007) }\end{array}$ & $\mathrm{F}$ & $\begin{array}{c}\sim 73 \\
(\mathrm{MZ816451)}\end{array}$ & $\begin{array}{c}\sim 63 \\
(\mathrm{MZ} 816494)\end{array}$ & $\begin{array}{c}\sim 261 \\
(\mathrm{MZ} 816538)\end{array}$ & $\begin{array}{c}\sim 269 \\
(\mathrm{MZ816587})\end{array}$ & $\begin{array}{c}410 \\
(\mathrm{MZ} 816640)\end{array}$ & $h^{S T}-5$ \\
\hline $\mathrm{i}-10$ & $\begin{array}{l}\text { Eclipophleps glacialis } \\
\text { (G, Altai, 2003) }\end{array}$ & B & 9 (MZ816482) & $\begin{array}{c}133 \\
(\mathrm{MZ} 816525)\end{array}$ & $\begin{array}{c}\sim 6 \mathrm{R} \\
(\mathrm{MZ} 816568)\end{array}$ & No & $\begin{array}{c}\sim 4 \\
(\mathrm{MZ} 816670)\end{array}$ & not full \\
\hline $\mathrm{i}-11$ & $\begin{array}{l}\text { Gomphocerippus rufus } \\
\text { (G, Novosibirsk, 2017) }\end{array}$ & B & 9 (MZ816452) & $\begin{array}{c}133 \\
(\mathrm{MZ} 816495)\end{array}$ & $\begin{array}{c}6 \\
(\mathrm{MZ} 816539)\end{array}$ & $\begin{array}{c}106 \\
(\mathrm{MZ} 816588)\end{array}$ & $\begin{array}{c}197 \\
(\mathrm{MZ816641})\end{array}$ & $\mathrm{h}^{\mathrm{ST}}-4$ \\
\hline $\mathrm{i}-12$ & $\begin{array}{l}\text { Omocestus viridulus } \\
\text { (Novosibirsk, 2009) }\end{array}$ & B & 9 (MZ816453) & $\begin{array}{c}133 \\
(\mathrm{MZ} 816496) \\
\end{array}$ & $\begin{array}{c}6 \\
(\mathrm{MZ} 816540) \\
\end{array}$ & $\begin{array}{c}106 \\
(\mathrm{MZ816589}) \\
\end{array}$ & $\begin{array}{c}197 \\
(\mathrm{MZ} 816642) \\
\end{array}$ & $\mathrm{h}^{\mathrm{ST}}-4$ \\
\hline i-13 & Om. Viridulus (Altai, 2017) & $\mathrm{B}$ & $\begin{array}{c}134 \\
(\mathrm{MZ} 816454)\end{array}$ & $\begin{array}{c}168 \\
(\mathrm{MZ} 816497)\end{array}$ & $\begin{array}{c}\sim 6 \\
(\mathrm{MZ} 816541)\end{array}$ & $\begin{array}{c}106 \\
(\mathrm{MZ} 816590)\end{array}$ & $\begin{array}{c}197 \\
(\mathrm{MZ} 816643)\end{array}$ & $\mathrm{h}^{\mathrm{ST}}-1$ \\
\hline $\mathrm{i}-14$ & $\begin{array}{l}\text { Om. Haemorrhoidalis } \\
\text { (Altai, 2017) }\end{array}$ & B & $\begin{array}{c}134 \\
(\mathrm{MZ} 816455)\end{array}$ & $\begin{array}{c}14 \\
(\mathrm{MZ} 816498)\end{array}$ & $\begin{array}{c}\sim 6 \\
(\mathrm{MZ} 816542)\end{array}$ & $\begin{array}{c}106 \\
(\mathrm{MZ} 816591)\end{array}$ & $\begin{array}{c}197 \\
(\mathrm{MZ} 816644)\end{array}$ & $\mathrm{h}^{\mathrm{ST}}-2$ \\
\hline $\mathrm{i}-15$ & $\begin{array}{l}\text { Podismopsis altaica } \\
\text { (Altai, 2003) }\end{array}$ & $\mathrm{F}$ & No & $\begin{array}{c}\sim 63 \\
(\mathrm{MZ} 816526) \\
\end{array}$ & $\begin{array}{c}\sim 325 \\
(\mathrm{MZ} 816569) \\
\end{array}$ & $?$ & $?$ & not full \\
\hline i-16 & $\begin{array}{l}\text { Podismopsis genicularibus } \\
\text { (Sakhalin Is., 2010) }\end{array}$ & B-A & $\begin{array}{c}134 \\
(\mathrm{MZ} 816456) \\
\end{array}$ & $\begin{array}{c}14 \\
(\mathrm{MZ} 816499)\end{array}$ & $\begin{array}{c}\sim 6 \\
(\mathrm{MZ} 816543)\end{array}$ & $\begin{array}{c}226 \\
(\mathrm{MZ} 816592) \\
\end{array}$ & $\begin{array}{c}197 \\
(\mathrm{MZ} 816645)\end{array}$ & $h^{S T}-6$ \\
\hline i-17 & $\begin{array}{l}\text { Pseudochorthippus montanus } \\
\text { (Novosibirsk, 2017) }\end{array}$ & B & 9 (MZ816457) & $\begin{array}{c}133 \\
(\mathrm{MZ} 816500)\end{array}$ & $\begin{array}{c}6 \\
(\mathrm{MZ} 816544) \\
\end{array}$ & $\begin{array}{c}106 \\
(\mathrm{MZ} 816593) \\
\end{array}$ & $\begin{array}{c}197 \\
(\mathrm{MZ} 816646) \\
\end{array}$ & $\mathrm{h}^{\mathrm{ST}}-4$ \\
\hline i-18 & $\begin{array}{c}\text { Ps. Montanus } \\
\text { (Novosibirsk, 2017) }\end{array}$ & B & 9 (MZ816483) & $\begin{array}{c}133 \\
(\mathrm{MZ816527)} \\
\end{array}$ & $\begin{array}{c}6 \\
(\mathrm{MZ} 816570) \\
\end{array}$ & $?$ & $?$ & not full \\
\hline i-19 & $\begin{array}{c}\text { Ps. Parallelus } \\
\text { (Novosibirsk, 2017) }\end{array}$ & B & 9 (MZ816458) & $\begin{array}{c}133 \\
(\mathrm{MZ} 816501)\end{array}$ & $\begin{array}{c}6 \\
(\mathrm{MZ} 816545) \\
\end{array}$ & $\begin{array}{c}106 \\
(\mathrm{MZ816594)}\end{array}$ & $\begin{array}{c}197 \\
(\mathrm{MZ} 816647)\end{array}$ & $\mathrm{h}^{\mathrm{ST}}-4$ \\
\hline $\mathrm{i}-20$ & $\begin{array}{l}\text { Stauroderus scalaris } \\
\text { (Altai, 2017) }\end{array}$ & B & 9 (MZ816459) & $\begin{array}{c}133 \\
(\mathrm{MZ} 816502) \\
\end{array}$ & $\begin{array}{c}6 \\
(\mathrm{MZ} 816546) \\
\end{array}$ & $\begin{array}{c}106 \\
(\mathrm{MZ816595)}\end{array}$ & $\begin{array}{c}197 \\
(\mathrm{MZ} 816648)\end{array}$ & $\mathrm{h}^{\mathrm{ST}}-4$ \\
\hline $\mathrm{i}-21$ & $\begin{array}{l}\text { Bryodema gebleri } \\
\text { (Altai, 2003) }\end{array}$ & $\mathrm{F}$ & $\begin{array}{c}\sim 73 \\
(\mathrm{MZ816460)}) \\
\end{array}$ & $\begin{array}{c}\sim 63 \\
(\mathrm{MZ} 816503) \\
\end{array}$ & $\begin{array}{c}\sim 261 \\
(\mathrm{MZ} 816547)\end{array}$ & $\begin{array}{c}\sim 205 \\
(\mathrm{MZ816596}) \\
\end{array}$ & $\begin{array}{c}410 \\
(\mathrm{MZ} 816649) \\
\end{array}$ & $\mathrm{h}^{\mathrm{ST}}-7$ \\
\hline $\mathrm{i}-22$ & $\begin{array}{l}\text { Bryodema gebleri } \\
\text { (Altai, 2017) }\end{array}$ & $\mathrm{F}$ & $\begin{array}{c}\sim 73 \\
(\mathrm{MZ816461)} \\
\end{array}$ & $\begin{array}{c}\sim 63 \\
(\mathrm{MZ816504)}) \\
\end{array}$ & $\begin{array}{c}\sim 261 \\
(\mathrm{MZ} 816548) \\
\end{array}$ & $\begin{array}{c}\sim 205 \\
(\mathrm{MZ816597}) \\
\end{array}$ & $\begin{array}{c}410 \\
(\mathrm{MZ} 816650) \\
\end{array}$ & $\mathrm{h}^{\mathrm{ST}}-7$ \\
\hline $\mathrm{i}-23$ & $\begin{array}{l}\text { Oedaleus decorus } \\
\text { (Altai, 2017) }\end{array}$ & $\mathrm{F}$ & $\begin{array}{c}\sim 73 \\
(\mathrm{MZ} 816462) \\
\end{array}$ & $\begin{array}{c}\sim 63 \\
(\mathrm{MZ} 816505) \\
\end{array}$ & $\begin{array}{c}\sim 35 \\
(\mathrm{MZ} 816549) \\
\end{array}$ & $\begin{array}{c}\sim 205 \\
(\mathrm{MZ816598)} \\
\end{array}$ & $\begin{array}{c}410 \\
(\mathrm{MZ} 816651) \\
\end{array}$ & $\mathrm{h}^{\mathrm{ST}}-8$ \\
\hline $\mathrm{i}-24$ & $\begin{array}{c}\text { Oe. Decorus } \\
\text { (Kazakhstan, 2006) }\end{array}$ & $\mathrm{F}$ & $\begin{array}{c}\sim 243 \\
(\mathrm{MZ} 816484)\end{array}$ & $\begin{array}{c}\sim 63 R K \\
(\mathrm{MZ} 816528)\end{array}$ & $\begin{array}{c}\sim 261 \\
(\mathrm{MZ} 816571)\end{array}$ & $\begin{array}{c}\sim 205 \mathrm{R} \\
(\mathrm{MZ816616})\end{array}$ & $\begin{array}{c}410 \\
(\mathrm{MZ} 816671)\end{array}$ & $\mathrm{N}$, full \\
\hline $\mathrm{i}-25$ & $\begin{array}{c}\text { Oe. Decorus } \\
\text { (Tajikistan, 2009) }\end{array}$ & $\mathrm{F}$ & $\begin{array}{c}\sim 243 \mathrm{Y} \\
(\mathrm{MZ} 816485) \\
\end{array}$ & $\begin{array}{c}\sim 30 \mathrm{YR} \\
(\mathrm{MZ816529})\end{array}$ & $\begin{array}{c}\sim 261 \mathrm{Y} \\
(\mathrm{MZ816572})\end{array}$ & $\begin{array}{c}\sim 205 \\
(\mathrm{MZ} 816617) \\
\end{array}$ & $\begin{array}{c}410 \\
(\mathrm{MZ} 816672)\end{array}$ & $\mathrm{N}$, full \\
\hline $\mathrm{i}-26$ & $\begin{array}{c}\text { Anapodisma miramae } \\
\text { (Far East, Russia, } 2008 \text { ) }\end{array}$ & B & 39 (MZ816463) & $\begin{array}{c}14 \\
(\mathrm{MZ} 816506)\end{array}$ & $\begin{array}{c}40 \\
(\mathrm{MZ} 816550)\end{array}$ & $\begin{array}{c}7 \\
(\mathrm{MZ816599})\end{array}$ & $\begin{array}{c}197 \\
(\mathrm{MZ816652})\end{array}$ & ST299 \\
\hline
\end{tabular}


Table 2. Cont.

\begin{tabular}{|c|c|c|c|c|c|c|c|c|}
\hline Isolate & Species (Region, Year) & Supergroup & gatB & $\operatorname{cox} \mathbf{A}$ & hсpA & ftsZ & $\mathrm{fbpA}$ & $\begin{array}{l}\text { Sequ-Ence } \\
\text { Type * }\end{array}$ \\
\hline$i-27$ & $\begin{array}{l}\text { Bohemanella frigida } \\
\text { (Altai 2003) }\end{array}$ & B & 9 (MZ816464) & $\begin{array}{c}9 \\
(\mathrm{MZ816507)}\end{array}$ & $\begin{array}{c}6 \\
(\mathrm{MZ816551)}\end{array}$ & $\begin{array}{c}106 \\
(\mathrm{MZ} 816600)\end{array}$ & $\begin{array}{c}197 \\
\text { (MZ816653) }\end{array}$ & $\mathrm{h}^{\mathrm{ST}}-9$ \\
\hline$i-28$ & $\begin{array}{l}\text { Ognevia longipennis } \\
\text { (Japan, 2005) }\end{array}$ & B & 9 (MZ816465) & $\begin{array}{c}133 \\
(\mathrm{MZ816508)}\end{array}$ & $\begin{array}{c}6 \\
(\mathrm{MZ816552)}\end{array}$ & $\begin{array}{c}106 \\
(\mathrm{MZ816601)}\end{array}$ & $\begin{array}{c}197 \\
(\mathrm{MZ} 816654)\end{array}$ & $\mathrm{h}^{\mathrm{ST}}-4$ \\
\hline$i-29$ & $\begin{array}{l}\text { Og. Longipennis } \\
\text { (Altai, 2003) }\end{array}$ & B & 9 (MZ816466) & $\begin{array}{c}133 \\
(\mathrm{MZ816509)}\end{array}$ & $\begin{array}{c}6 \\
(\mathrm{MZ816553)}\end{array}$ & $\begin{array}{c}106 \\
(\mathrm{MZ816602)}\end{array}$ & $\begin{array}{c}197 \\
(\mathrm{MZ} 816655)\end{array}$ & $\mathrm{h}^{\mathrm{ST}}-4$ \\
\hline$i-30$ & $\begin{array}{l}\text { Podisma pedestris } \\
\text { (Altai, 2003) }\end{array}$ & B & 9 (MZ816467) & $\begin{array}{c}133 \\
(\mathrm{MZ816510)}\end{array}$ & $\begin{array}{c}6 \\
(\mathrm{MZ816554)}\end{array}$ & $\begin{array}{c}106 \\
(\mathrm{MZ816603)}\end{array}$ & $\begin{array}{c}197 \\
(\mathrm{MZ816656)}\end{array}$ & $\mathrm{h}^{\mathrm{ST}}-4$ \\
\hline$i-31$ & P. pedestris (Altai, 2016) & B & 9 (MZ816468) & $\begin{array}{c}133 \\
(\mathrm{MZ816511})\end{array}$ & $\begin{array}{c}6 \\
(\mathrm{MZ816555)}\end{array}$ & $\begin{array}{c}106 \\
(\mathrm{MZ816604)}\end{array}$ & $\begin{array}{c}197 \\
(\mathrm{MZ816657)}\end{array}$ & $\mathrm{h}^{\mathrm{ST}}-4$ \\
\hline$i-32$ & $\begin{array}{l}\text { Prumna littoralis (Far East, } \\
\text { Russia, 2008) }\end{array}$ & B & 9 (MZ816469) & $\begin{array}{c}133 \\
(\mathrm{MZ816512})\end{array}$ & $\begin{array}{c}6 \\
(\mathrm{MZ816556)} \\
\end{array}$ & $\begin{array}{c}106 \\
(\mathrm{MZ816605)}\end{array}$ & $\begin{array}{c}197 \\
(\mathrm{MZ816658)}\end{array}$ & $\mathrm{h}^{\mathrm{ST}}-4$ \\
\hline$i-33$ & Pr. Primnoa (Sakhalin, 2010) & B & 9 (MZ816470) & $\begin{array}{c}133 \\
(\mathrm{MZ816513)} \\
\end{array}$ & $\begin{array}{c}6 \\
(\mathrm{MZ816557)} \\
\end{array}$ & $\begin{array}{c}106 \\
(\mathrm{MZ816606)} \\
\end{array}$ & $\begin{array}{c}197 \\
(\mathrm{MZ816659)} \\
\end{array}$ & $\mathrm{h}^{\mathrm{ST}}-4$ \\
\hline$i-34$ & $\begin{array}{c}\text { Pr. Primnoa } \\
\text { (Sakhalin Is., 2010) }\end{array}$ & B & $\begin{array}{c}188 \\
(\mathrm{MZ816471)}\end{array}$ & $\begin{array}{c}224 \\
(\mathrm{MZ} 816514)\end{array}$ & $\begin{array}{c}\sim 6 \\
(\mathrm{MZ816558)}\end{array}$ & $\begin{array}{c}20 \\
(\mathrm{MZ} 816607) \\
\end{array}$ & $\begin{array}{c}25 \\
(\mathrm{MZ} 816660) \\
\end{array}$ & $h^{\mathrm{ST}}-10$ \\
\hline$i-35$ & $\begin{array}{l}\text { Pr. Ussuriensis (Far East, } \\
\text { Russia, 2008) }\end{array}$ & B & 9 (MZ816472) & $\begin{array}{c}133 \\
(\mathrm{MZ816515)} \\
\end{array}$ & $\begin{array}{c}6 \\
(\mathrm{MZ816559)} \\
\end{array}$ & $\begin{array}{c}106 \\
(\mathrm{MZ816608)}\end{array}$ & $\begin{array}{c}197 \\
(\mathrm{MZ} 816661) \\
\end{array}$ & $h^{\mathrm{ST}}-4$ \\
\hline$i-36$ & $\begin{array}{l}\text { Podisma kanoi (Honshu, } \\
\text { Japan, 2005) }\end{array}$ & B & 9 (MZ816473) & $\begin{array}{c}133 \\
(\mathrm{MZ816516)}\end{array}$ & $\begin{array}{c}6 \\
(\mathrm{MZ} 816560) \\
\end{array}$ & $\begin{array}{c}106 \\
(\mathrm{MZ} 816609)\end{array}$ & $\begin{array}{c}197 \\
(\mathrm{MZ} 816662) \\
\end{array}$ & $\mathrm{h}^{\mathrm{ST}}-4$ \\
\hline $\mathrm{i}-37$ & $\begin{array}{c}\text { P. sapporensis } \\
\text { (Japan, Tanno, 2005) }\end{array}$ & B & 9 (MZ816474) & $\begin{array}{c}14 \\
(\mathrm{MZ816517}) \\
\end{array}$ & $\begin{array}{c}6 \\
(\mathrm{MZ816561)} \\
\end{array}$ & $\begin{array}{c}106 \\
(\mathrm{MZ} 816610)\end{array}$ & $\begin{array}{c}197 \\
(\mathrm{MZ} 816663) \\
\end{array}$ & $\mathrm{h}^{\mathrm{ST}}-11$ \\
\hline $\mathrm{i}-38$ & $\begin{array}{c}\text { P. sapporensis } \\
\text { (Japan, Akan, 2005) }\end{array}$ & B & 9 (MZ816475) & $\begin{array}{c}14 \\
(\mathrm{MZ} 816518) \\
\end{array}$ & $\begin{array}{c}6 \\
(\mathrm{MZ} 816562) \\
\end{array}$ & $\begin{array}{c}106 \\
(\mathrm{MZ} 816611) \\
\end{array}$ & $\begin{array}{c}197 \\
(\mathrm{MZ} 816664) \\
\end{array}$ & $h^{\mathrm{ST}}-11$ \\
\hline$i-39$ & $\begin{array}{c}\text { P. sapporensis } \\
\text { (Japan, Yotei, 2005) }\end{array}$ & B & 9 (MZ816476) & $\begin{array}{c}14 \\
(\mathrm{MZ816519)} \\
\end{array}$ & $\begin{array}{c}6 \\
(\mathrm{MZ} 816563) \\
\end{array}$ & $\begin{array}{c}106 \\
(\mathrm{MZ} 816612)\end{array}$ & $\begin{array}{c}197 \\
(\mathrm{MZ} 816665) \\
\end{array}$ & $h^{\mathrm{ST}}-11$ \\
\hline$i-40$ & $\begin{array}{l}\text { P. sapporensis (Japan, } \\
\text { Disengen, 2005) }\end{array}$ & B & 9 (MZ816486) & $\begin{array}{c}14 \\
(\mathrm{MZ816530)}\end{array}$ & $\begin{array}{c}6 \\
(\mathrm{MZ} 816573)\end{array}$ & $\begin{array}{l}\text { Mix ***** } \\
\text { (MZ816618- } \\
\text { MZ816632) }\end{array}$ & $\begin{array}{c}197 \\
(\mathrm{MZ} 816673)\end{array}$ & Mix \\
\hline$i-41$ & $\begin{array}{l}\text { P. sapporensis (Japan, } \\
\text { Naganuma, 2005) }\end{array}$ & B & 9 (MZ816477) & $\begin{array}{c}14 \\
(\mathrm{MZ816520)}\end{array}$ & $\begin{array}{c}6 \\
(\mathrm{MZ816564)}\end{array}$ & $\begin{array}{c}106 \\
(\mathrm{MZ816613)}\end{array}$ & $\begin{array}{c}197 \\
(\mathrm{MZ816666)}\end{array}$ & $h^{\mathrm{ST}}-11$ \\
\hline $\mathrm{i}-42$ & $\begin{array}{c}\text { P. sapporensis } \\
\text { (Japan, Teine, 2005) }\end{array}$ & B & 9 (MZ816487) & $\begin{array}{c}73 \\
(\mathrm{MZ} 816531)\end{array}$ & $\begin{array}{c}\text { Mix } \\
\text { (MZ816574- } \\
\text { MZ816580) }\end{array}$ & $\begin{array}{c}106 \\
(\mathrm{MZ} 816633)\end{array}$ & $\begin{array}{c}\text { Mix } \\
\text { (MZ816674- } \\
\text { MZ816686) }\end{array}$ & Mix \\
\hline$i-43$ & $\begin{array}{l}\text { P. sapporensis (Japan, } \\
\text { Shimokawa, 2005) }\end{array}$ & B & 9 (MZ816478) & $\begin{array}{c}14 \\
(\mathrm{MZ} 816521)\end{array}$ & $\begin{array}{c}6 \\
(M Z 816565)\end{array}$ & $\begin{array}{c}106 \\
(\mathrm{MZ} 816614)\end{array}$ & $\begin{array}{c}197 \\
(\mathrm{MZ} 816667)\end{array}$ & $h^{\mathrm{ST}}-11$ \\
\hline $\mathrm{i}-44$ & $\begin{array}{l}\text { P. tyatiensis (Kunashir Is., } \\
\text { Russia, 2001) }\end{array}$ & B & 9 (MZ816479) & $\begin{array}{c}14 \\
(\mathrm{MZ} 816522)\end{array}$ & $\begin{array}{c}6 \\
(\mathrm{MZ} 816566)\end{array}$ & $\begin{array}{c}106 \\
(\mathrm{MZ} 816615)\end{array}$ & $\begin{array}{c}197 \\
(\mathrm{MZ} 816668)\end{array}$ & $h^{\mathrm{ST}}-11$ \\
\hline
\end{tabular}

*ST numbers according to the PubMLST database or haplotype numbers according to this study or short comments are provided; ${ }^{* *}$ the ' $\sim$ number' refers to the most closely related alleles according to the PubMLST database; ${ }^{* * *}$ no PCR product; ${ }^{* * * *}$ multiple double chromatogram peaks; ${ }^{* * * *}$ mix: a DNA sample that yielded multiple double chromatogram peaks was cloned and sequenced.

We reconstructed ML phylogenetic trees for each locus to examine supergroup clustering (Figure 1, Supplementary Materials). Alleles of the analysed isolates belong to Wolbachia supergroups B and F. In particular, species belonging to Podisminae only harboured variants from supergroup $B$, species belonging to Oedipodinae only harboured variants from supergroup $\mathrm{F}$, and species belonging to Gomphocerinae mostly harboured species from super group B with some from supergroup F. Overall, the genetic diversity of the MLST loci appeared rather low (Table 2; Figures 1 and 2, Supplementary Materials). This result was especially evident at the $f b p A$ locus, where nearly all of the B-supergroup isolates contained the $f b p A-197$ allele and where all of the F-supergroup isolates contained fbpA-410. Moreover, there was one case of an inter-supergroup recombination. A complete haplotype of i-16 
(Podismopsis genicularibus) was found to be basal to supergroup B because the ftsZ-226 allele belonged to supergroup A, whereas alleles from other loci were assigned to supergroup B.
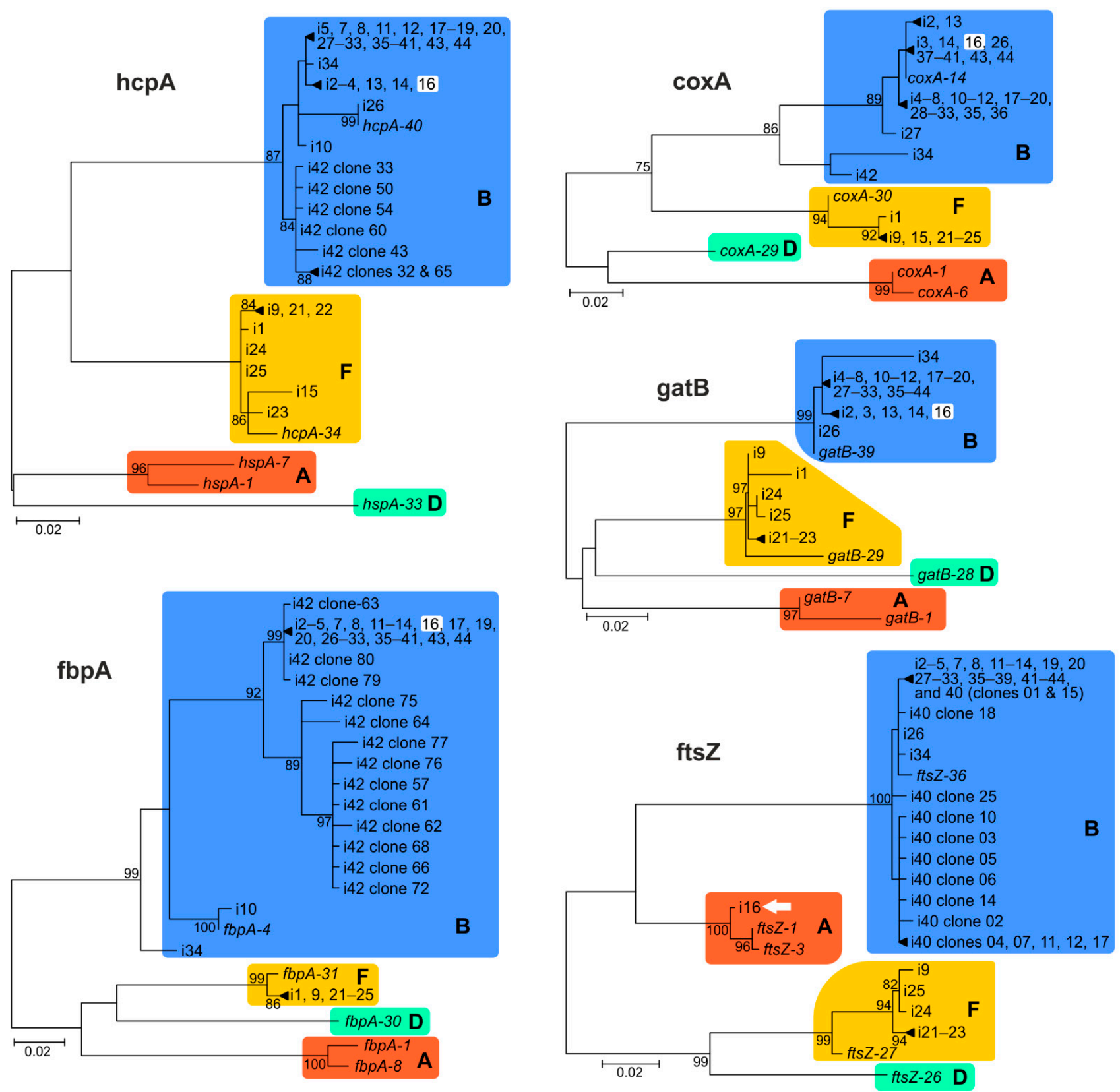

Figure 1. Maximum likelihood (ML) phylogenetic trees for each MLST gene. Acrididae isolates (Table 2), supergroups (A, B, F, and D), and bootstrap values are indicated. Model of nucleotide substitutions $\mathrm{T} 92+\mathrm{G}$ was used for $g a t B, h c p A$, and $f b p A$ datasets; $\mathrm{HKY}+\mathrm{G}$ for coxA; T92+G for $f t s Z$. White squares and the arrow indicate a case (i-16) of a supergroup clustering conflict. See original files in Supplementary Materials. 


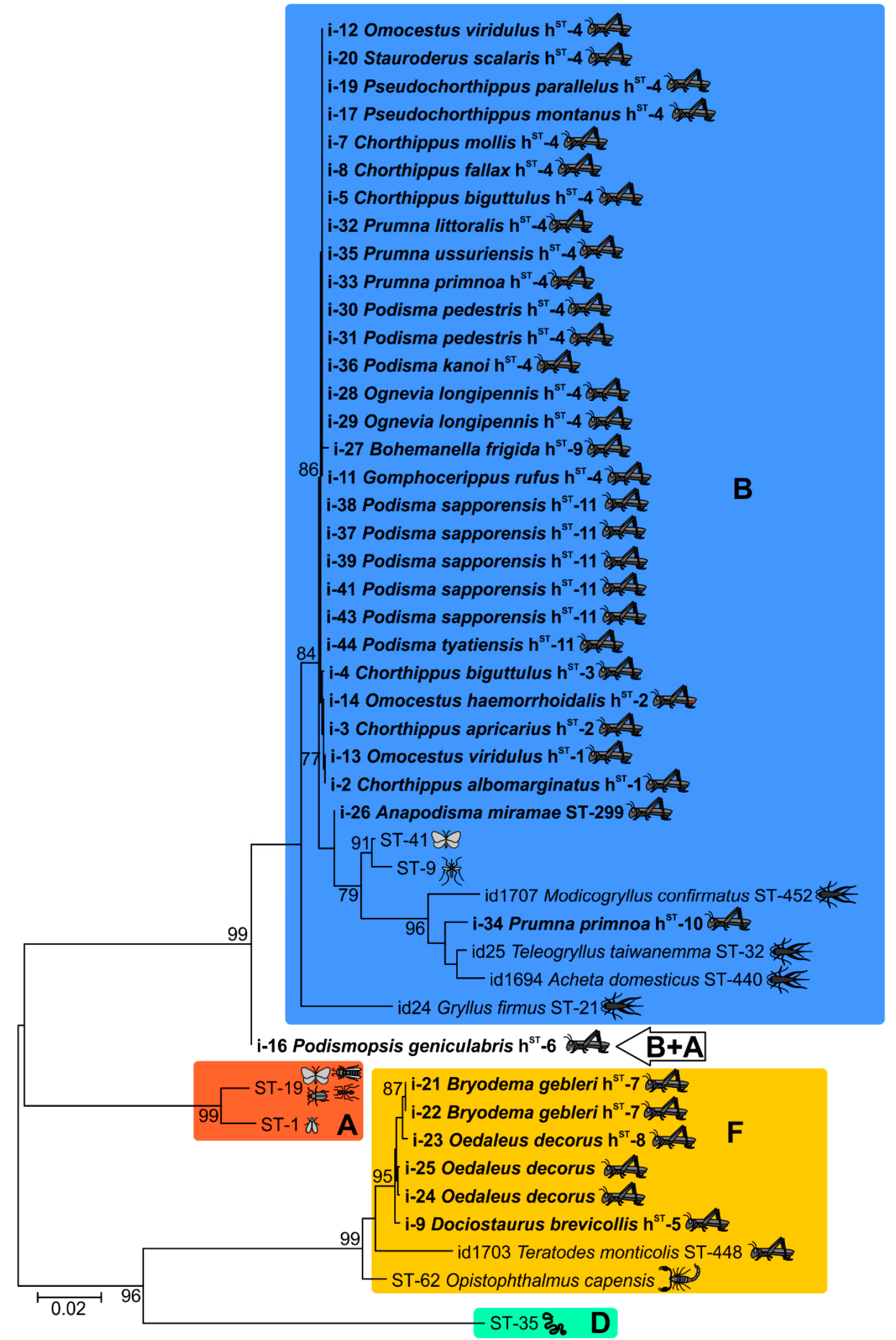

Figure 2. An ML phylogenetic tree of Wolbachia symbionts isolated from Acrididae hosts. The dataset is based on the concatenation of five genes of the MLST protocol, model T92+G, and bootstrapping with 1000 iterations (values higher than 75 are provided). See original files in Supplementary Materials. 
Only haplotype ST-299 has been reported upon earlier [46], whereas others turned out to be unique because of new alleles or new allele combinations. A new combination of previously known alleles ( $g a t B-9, \operatorname{cox} A-133, h c p A-6, f t s Z-106$, and $f b p A-197)$ that we designated as ST $\mathrm{h}^{\mathrm{ST}}-4$ (see Materials and Methods) was the most frequent in the study population; it was present in seven species of Gomphocerinae and in six species of Podisminae. Haplotypes $h^{\mathrm{ST}}-1,-2,-3,-9$, and -11 , which are closely related to $\mathrm{h}^{\mathrm{ST}}-4$ ( $p$-distance 0.0005-0.0020, namely, 1-4 mutations), were also revealed in Gomphocerinae and Podisminae species in geographically distant populations. These six haplotypes form an ${ }^{\mathrm{ST}}-4$ group' that includes a constant allele set of ftsZ-106 and fbpA-197 as well as varied but closely related (to each other) alleles of $g a t B, \operatorname{cox} A$, and $h c p A$ loci. Other B-supergroup haplotypes were distantly related to $\mathrm{h}^{\mathrm{ST}}-4$ ( $p$-distance for ST-299: 0.0058, for $\mathrm{h}^{\mathrm{ST}}-6$ : 0.0203 and for $\mathrm{h}^{\mathrm{ST}}-10$ : 0.0323); however, they shared identical alleles with the $\mathrm{h}^{\mathrm{ST}}-4$ group. These phenomena can be explained by intra-supergroup recombination.

Wolbachia strains belonging to supergroup F were detected in five species. Haplotypes of complete profiles $\left(\mathrm{h}^{\mathrm{ST}}-5,-7\right.$, and -8; $p$-distance $\left.0.0024-0.0053\right)$ and isolates with incomplete profiles (i-1 and i-15) or with ambiguous sites (i-24 and i-25) were found to be closely related, i.e., genetic diversity was also rather low. A comparative analysis of the haplotype diversity of supergroup F retrieved from the PubMLST database showed that variants of the Acrididae isolates formed a separate cluster (Figure S1).

To examine the relationship of the Wolbachia variants isolated from Acrididae with other Wolbachia strains, we reconstructed a phylogenetic network (Figure 3, Supplementary Materials). The dataset that was used for this analysis included Wolbachia haplotypes from different hosts that contained at least one allele that was identical or that was closely related to Wolbachia isolates from Acrididae (see Materials and Methods). Most Wolbachia isolates of Acrididae (actually the $\mathrm{h}^{\mathrm{ST}}-4$ group) formed a separate bundle in the phylogenetic network without isolates from other hosts. Even i-16, which is an inter-supergroup recombinant, occupied a long branch in this bundle. The remaining Wolbachia haplotype $\mathrm{h}^{\mathrm{ST}}-10$ (i-34) manifested a close relationship with isolates from Gryllidae hosts. Of note, i-34 was isolated from the Prumna primnoa population of Sakhalin in 2010, where the $\mathrm{h}^{\mathrm{ST}}-4$ (i-33) haplotype was identified as well. Haplotypes of the F supergroup also formed a separate bundle. Only the F-supergroup Wolbachia haplotype ST-448 isolated from Teratodes monticollis (Acrididae: Teratodinae) was noticeably different in the allele set and genetic distance ( $p$-distance 0.0293-0.0313). Therefore, the additional data from the MLST profiles based on allele similarity confirmed that the analysed Acrididae subfamilies have a specific genetic pattern of Wolbachia.

Martinez-Rodriguez and Bella [44] reported the Wolbachia MLST diversity of Ch. parallelus in Spanish and French locations. We retrieved sequences of five MLST loci deposited by [44] in GenBank, combined them with the dataset of our phylogenetic network, excluded redundant portions of sequences, and reconstructed a new phylogenetic network (Figure 4, Supplementary Materials). Half of the Wolbachia variants from Ch. parallelus matched the genetic pattern discovered in our collection for both the B- and F-supergroup haplotypes. The other half represented B-F supergroup recombinants. B-F recombinants were registered by [44]; here, we reported detailed characteristics of some variants (details in Figure 4). It is worth pointing out that the recombination occurred at all possible loci, thereby giving rise to 18 unique haplotypes in total. 


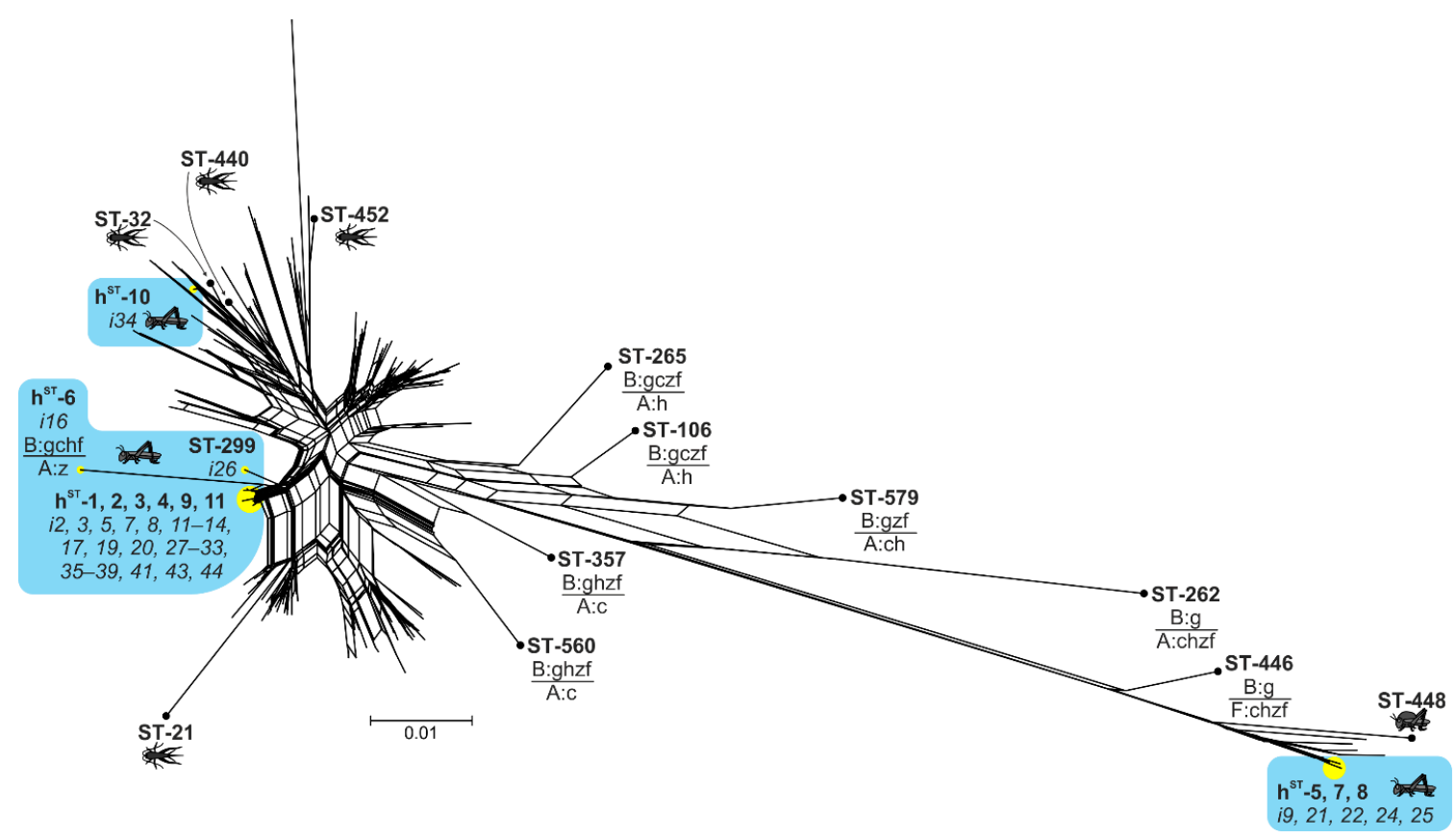

Figure 3. The phylogenetic network (NeighborNet) constructed in SplitsTree4 from 215 unique haplotypes (concatenated MLST genes). Branches with isolates from this study are highlighted in yellow. Other haplotype profiles were included here because (1) an Orthoptera host (insect symbol provided) or (2) a haplotype shared at least one identical or closely related allele with the studied Acrididae isolates. Inter-supergroup recombinant haplotypes are designated as 'supergroup (A, B or F): genes g (gatB), $\mathrm{c}(\operatorname{coxA}), \mathrm{h}(\mathrm{hcp} A), \mathrm{z}(\mathrm{ftsZ}), \mathrm{f}(\mathrm{fbpA})^{\prime}$. See original files in Supplementary Materials.

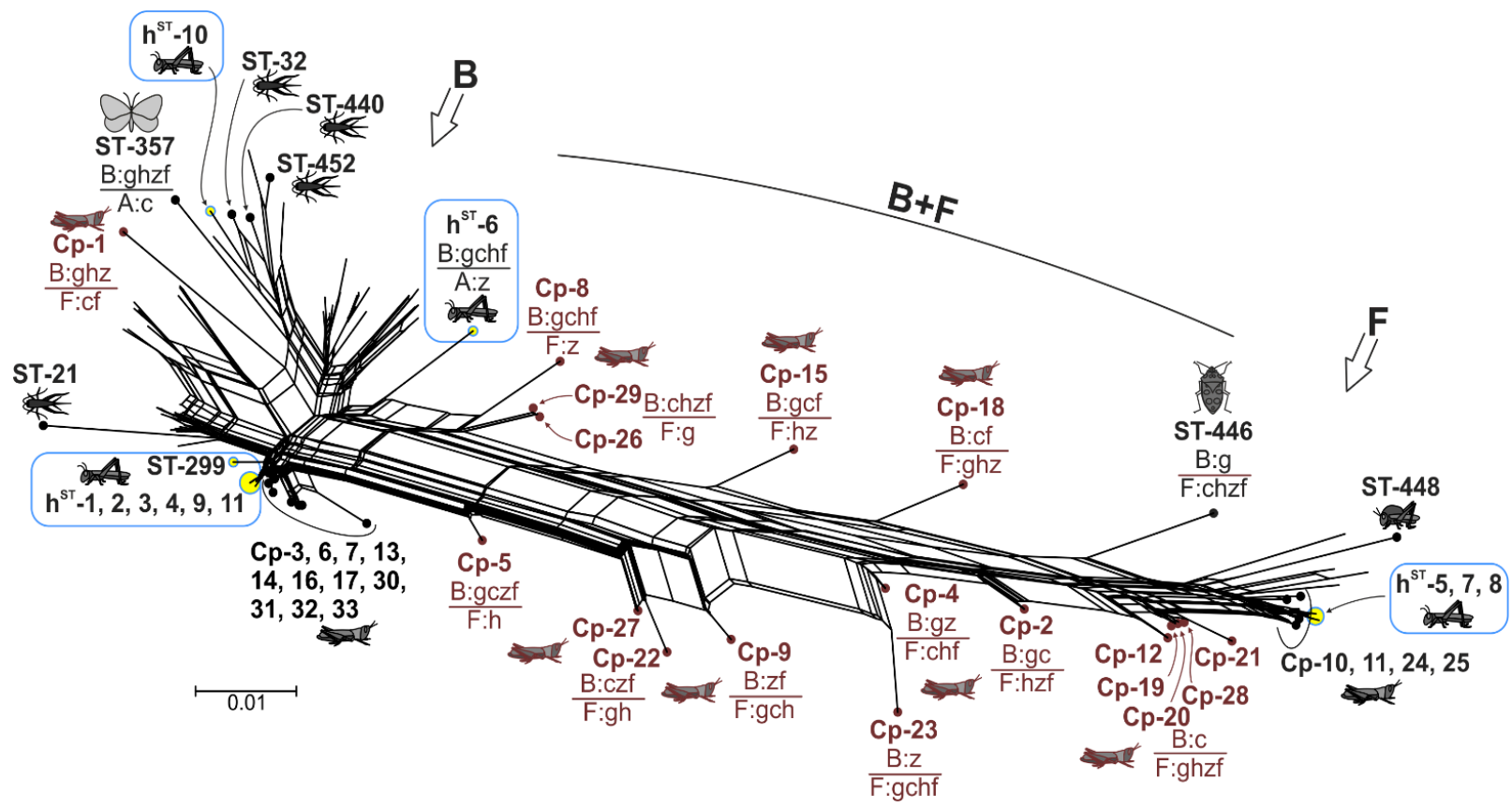

Figure 4. The phylogenetic network (NeighborNet) constructed in SplitsTree4 from 127 unique haplotypes (concatenated MLST genes). Branches with isolates from this study are highlighted in yellow, isolates from [44] (Cp) are in brown. The dataset of this figure differs from that in Figure 3 in the following ways: (1) the number of haplotypes was reduced (to decrease phylogenetic noise), (2) the alignment corresponds to haplotype data from [44]. Inter-supergroup recombinant haplotypes are designated as in Figure 3. See original files in Supplementary Materials. 


\subsection{Incomplete MLST Profiles}

As mentioned above, for some isolates, we could not obtain an amplicon or unambiguous sequences. Amplicons of three such cases in two isolates of P. sapporensis (i-40 and i-42) were cloned and sequenced. Fifteen clones of an fts $Z$ amplicon of i-40 represented 11 variants; among them, we regard two variants-ftsZ-106 (two clones) and ftsZ-81 with substitution $\mathrm{A}^{408}$ (five clones) - as authentic (see the last sentence of this subsection) because the former is commonly found in other P. sapporensis isolates, and the latter one was assembled manually after direct amplicon sequencing. The other eight variants represented by unique clones were close to the alleles ftsZ-7, -81 , and -106 , but they differed by amino acid substitutions, and there was even a stop codon in clone 5. A similar pattern was observed during the screening of the $h c p A$ and $f b p A$ amplicons of $\mathrm{i}-42$. There were seven variants that were closely related to the allele $h c p A-142$ with 4-7 mutations, including the variant $h c p A-142 \mathrm{G}^{298} \mathrm{~A}^{373} \mathrm{G}^{393} \mathrm{~T}^{438}$, which was manually assembled after direct amplicon sequencing. In the case of $f b p A$ cloning, one variant matched $f b p A-197$, two differed by one mutation, and the others differed by 13-19 mutations. Therefore, the cloning of the PCR products revealed several variants: ftsZ-106, ftsZ-81 A $\mathrm{A}^{408}$, and $h c p A-142 \mathrm{G}^{298} \mathrm{~A}^{373} \mathrm{G}^{393} \mathrm{~T}^{438}$ in i-40 as well as $f b p A-197$ and probably one mutant variant of $f b p A-197$ in $\mathrm{i}-42$. They most likely characterise the Wolbachia genome, whereas other variants quite possibly reflect Taq polymerase artefacts or denote Wolbachia genome segments that became integrated into the host genome.

\section{Discussion}

The Acrididae family includes more than 10 thousand species inhabiting different climatic zones from tropical rainforests to the subarctic zone. There is extensive information about the geographical distribution and economic significance of this group of insects and about the factors affecting the regulation of grasshopper population size in natural and anthropogenic landscapes. Nevertheless, knowledge about the symbionts of this group of insects remains meagre. Here, we presented the most detailed survey of Wolbachia diversity in Acrididae to date; 28 species out of 40 appeared to be infected by Wolbachia. On the other hand, this list, together with other studies $[23,32-37,44]$, comprises less than $1 \%$ of known Acrididae species. To achieve a more informative picture of Wolbachia genetic diversity in this family, it is necessary to examine other big subfamilies, and special attention should be paid to collections from warm climate zones, where the diversity is especially high. Currently, our results indicate that Wolbachia infection (i) is widespread among three subfamilies of Acrididae grasshoppers, (ii) can reach high prevalence in populations, and (iii) can be detected by means of DNA isolated from somatic tissues.

Our main result is the narrow genetic diversity of Wolbachia variants in Acrididae hosts. While Wolbachia in insects is commonly represented by the strains of supergroups A and B, the Acrididae hosts proved to be infected with supergroups B and F without A-supergroup variants. The only trace of an A-supergroup lineage was found in i-16, where the haplotype $\mathrm{h}^{\mathrm{ST}}-5$ includes the fts $\mathrm{Z}$ gene from supergroup A and other genes from the B supergroup. The variation of the B-supergroup haplotypes in Acrididae is especially low, as clearly illustrated by the phylogenetic network (Figure 3), where all the haplotypes included in the analysis showed allelic similarity with grasshopper Wolbachia. Previously, an allele set (with central haplotype ST-41) specific to butterfly hosts was characterised [46]; however, the Wolbachia variation observed here in Acrididae is much lower. The accumulation of data on Wolbachia infection in Acrididae hosts will possibly shed light on other Wolbachia variants, including A-supergroup variants. Nonetheless, it is already obvious that Acrididae hosts have a unique infection profile in the global pattern of the Wolbachia pandemic.

Active horizontal transmission (HT) of a specific set of Wolbachia strains among Acrididae grasshoppers is a reason for the observed narrow diversity. Here, we documented (i) cases (often seen in Wolbachia studies) where the same haplotype is found in different species (Table 2), (ii) findings of identical haplotype profiles in populations of Ognevia longipennis from Japan and Altai Mountains of Russia even though these populations most 
likely have been geographically isolated for several thousand years, and (iii) distantly related Wolbachia haplotypes within the Sakhalin population of P. primnoa (Sakhalin 2010). There is no clear understanding of the HT mechanism, although some data have been reported $[22,25,26,29,47]$. Potential vectors of Wolbachia HT are parasitoid wasps, red velvet mites, tachinids, entomophilic nematodes, and horsehair worms (Nematomorpha) that have wider or narrower specificity to Acrididae hosts. The next question is the nature of Wolbachia specificity to hosts. Are Acrididae species only susceptible to certain variants of Wolbachia, or does the susceptibility extend to all encountered variants?

The Wolbachia allelic diversity observed in our study is consistent with the findings about Wolbachia in Ch. parallelus studied on the Perinea peninsula [44]. A comprehensive analysis of the data generated by [44] and our results indicates that Wolbachia variants belonging to supergroups B and F are widespread in Acrididae hosts. Moreover, Ch. parallelus was found to harbour nearly every possible MLST recombinant combination of supergroups B and F (Figure 4). In our work, we were only able to reliably detect one isolate of inter-supergroup recombination. In five isolates, we obtained ambiguous signals in chromatograms. The nature of the problem for three of them (i-6, -15, and -18) was not investigated here, i.e., co-infection with B and F lineages cannot be ruled out. Two other isolates were cloned, and we came to the conclusion that the results can be explained by multi-infection with B-supergroup strains and/or by insertions of Wolbachia genes into the host's nuclear genome. The transfer of Wolbachia genes into a host nuclear genome in insects has been well documented [48-52], e.g., in Orthoptera [42,43].

Another essential issue of Wolbachia diversity is the concept of a bacterial species [53-55]. Every Wolbachia supergroup is a species candidate [6,56-59]. This observation implies genome specificity, ecology specificity, and 'reproductive isolation', which in bacteria, take the form of a low rate or impossibility of gene exchange between strains of different supergroups/species. Indeed, recombination between strains of supergroups A and B is known to occur, albeit at a low rate [46,57,60-63]. Recombination between supergroups $F$ and $B$ has been only detected in Chrysocoris stollii (Hemiptera) [62]; however, in the case of Ch. parallelus, there are 18 haplotypes [44]. Comprehensive genomic analysis of core genes indicates that the $\mathrm{F}$ supergroup clusters together with $\mathrm{C}$ and $\mathrm{D}$ lineages found in nematode hosts $[63,64]$. The F lineage has retained the genes responsible for homologous recombination (data not shown); we concluded this after the examination of the wCle genome (GenBank accession No.: AP013028). Previously, the evolution of these genes was studied in the genomes belonging to supergroups A, B, C, and D [65] but not in F. These data suggest that gene exchange between the genomes of $\mathrm{B}$ and $\mathrm{F}$ strains in $\mathrm{Ch}$. parallelus may occur via homologous recombination. If rampant recombination has actually occurred in Ch. parallelus, it casts serious doubt on the idea that supergroups B and F can be considered independent bacterial species.

\section{Materials and Methods}

\subsection{Collection of Specimens}

Grasshopper specimens were collected from natural populations during the period of 2001-2017 (Table 1). The specimens were fixed in 96\% ethanol and were stored at $-20{ }^{\circ} \mathrm{C}$. The total study population included 501 specimens from Gomphocerinae, Oedipodinae, and Podisminae, with four species being predominant (64.3\%): Chorthippus biguttulus (215), Chorthippus fallax (17), P. sapporensis (35), and Pseudochorthippus montanus (50).

\subsection{Screening and Sequencing}

A leg of an individual was used for DNA extraction in most cases. This approach is rather convenient because it allows the procedure to be repeated in cases of failed extraction (just take another leg) and to avoid bacterial contamination from the digestive system due to the facultative predation/cannibalism of Orthoptera species. Nonetheless, the use of somatic tissues does not permit a reliable estimation of Wolbachia prevalence in a population. Male gonad tissues were used for the screening of Podisma species collected in Hokkaido (Japan) and Kunashir (Russia), which were partially reported by Bugrov et al. [33]. Here, we added 
18 specimens and present full MLST profiles for the Podisma spp. hosts. DNA extraction from each sample was performed in $0.3-0.6 \mathrm{~mL}$ of extraction buffer $(0.1 \mathrm{M} \mathrm{NaCl}, 10 \mathrm{mM}$ Tris$\mathrm{HCl}$ (pH8.0), $25 \mathrm{mM}$ EDTA, 0.5\% SDS, and $0.1 \mathrm{mg} / \mathrm{mL}$ proteinase $\mathrm{K}$ ) for $2 \mathrm{~h}$ at $+56{ }^{\circ} \mathrm{C}$, and DNA was then salted out with $0.5 \mathrm{~V}$ of $5 \mathrm{M}$ potassium acetate/3 $\mathrm{M}$ acetic acid, after which DNA was further precipitated and dissolved in $0.2 \mathrm{~mL}$ of double-distillated $\mathrm{H}_{2} \mathrm{O}$. Next, $1 \mu \mathrm{L}$ of the DNA solution was used in all polymerase chain reactions (PCRs). The quality of the DNA was checked with universal primers specific to the nuclear gene of 28S rRNA (28sF3633: 5'-TACCGTGAGGGAAAGTTGAAA-3' , and 28sR4076: 5'-AGACTCCTTGGTCCGTGTTT$3^{\prime}$ [66]) or to mitochondrial gene CO1 (LCO1490: 5'-GGTCAACAAATCATAAAGATATTGG3', and HCO2198: 5' - TAAACTTCAGGGTGACCAAAAAATCA-3' [67]). PCR was conducted using BioMaster HS-Taq PCR $(2 \times)$ (BiolabMix, Novosibirsk, Russia) or a mix containing $3.0 \mathrm{mM} \mathrm{Mg}{ }^{2+}, 0.6 \mathrm{mM}$ each primer, $1 \times$ PCR buffer $\left(16 \mathrm{mM}\left(\mathrm{NH}_{4}\right)_{2} \mathrm{SO}_{4}, 67 \mathrm{mM}\right.$ Tris- $\mathrm{HCl}$ $\mathrm{pH} 8.8$ (at $25^{\circ} \mathrm{C}$ ), and $0.1 \%$ of Tween 20$)$, and $1.0 \mathrm{U}$ of Taq polymerase in a total reaction volume of $20 \mu \mathrm{L}$. The detection of Wolbachia infection in each specimen was performed by means of at least two MLST loci, as usually done with primer sets coxAF1/R1 and ftsZF1/R1 [12]. All five MLST loci were amplified and sequenced for Wolbachia-positive DNA samples. In cases of a weak amplicon signal or negative PCR results, the nested-PCR approach was employed [68]. External primer sets F2/R2 or F3/R3 were used for $g a t B, \operatorname{cox} A, h c p A$, and $f b p A$ loci according to [https:/ / pubmlst.org/organisms/wolbachia-spp/protocol-single-infected, accessed on 12 December 2021], primers ftsZunif1/2 for the ftsZ locus according to [6]; and the inner primers F1/R1 according to [12]. The thermal cycling conditions were as follows: initial denaturation at $95^{\circ} \mathrm{C} 5 \mathrm{~min}$, followed by 35 cycles of conventional PCR, and $15+30$ cycles of nested PCR at $95{ }^{\circ} \mathrm{C}$ for $15 \mathrm{~s}$, annealing at $55^{\circ} \mathrm{C}$ for the MLST primers or at $58{ }^{\circ} \mathrm{C}$ for $28 \mathrm{~S}$ or at $53{ }^{\circ} \mathrm{C}$ for $\mathrm{CO} 1$ for $40 \mathrm{~s}$, elongation at $72{ }^{\circ} \mathrm{C} 30 \mathrm{~s}-1 \mathrm{~min}$, and final elongation for $3 \mathrm{~min}$. In the second round of nested PCR, we added $0.5 \mu \mathrm{L}$ of the reaction mixture from the first round. The PCR products were visualised by agarose gel (1.0-1.5\%) electrophoresis with ethidium bromide. A portion of the amplification reaction mixture was diluted as follows: $2 \mu \mathrm{L}$ of the amplicon $+18 \mu \mathrm{L}$ of water; then $1 \mu \mathrm{L}$ of this solution was treated with $10 \mathrm{U}$ of exonuclease I (New England Biolabs, Ipswich, MA, USA) in the supplied buffer and sequenced using the BrightDye Terminator Cycle Sequencing Kit (Nimagen, Nijmegen, The Netherlands) or BigDye Terminator v3.1 cycle sequencing Kit (Applied Biosystems, Foster City, CA, USA). Three amplicons (ftsZ of i-40; $h c p A$ and $f b p A$ of i-42, which yielded ambiguous sequences) were cloned in the pAL-TA vector (Evrogen, Moscow, Russia) according to the manufacturer's instructions and were sequenced with the M13 primer set. The MLST profiles of the Wolbachia isolates were deposited in the GenBank database under accession numbers MZ816445-MZ816686. Because the MLST database has not been accepting new submissions for some time, here we designated haplotypes with new combinations of alleles or new alleles as ' $\mathrm{h}$ ST-Number' (Table 2).

\subsection{Evolutionary Analysis}

New MLST loci sequences were checked for stop codons, and sequence length was limited according to the MLST protocol for subsequent allele analysis. All of the sequences were used to reconstruct maximum likelihood (ML) phylogenetic trees of each MLST locus. As a supergroup reference, we used the alleles that had been retrieved from the following sequence types (STs): ST-1 (supergroup A), ST-19 (A), ST-41 (B), ST-35 (D), and ST-62 (supergroup F). Moreover, we reconstructed an ML phylogenetic tree of concatenated sequences for isolates with complete MLST profiles. A set of STs that represented supergroups A (ST-1 and ST-19), B (ST-9 and ST-41), D (ST-35), and F (ST-62) and set of STs that had been previously isolated from Orthoptera hosts (id-24 (ST-21), id-25 (ST-32), id-1694 (ST-440), id-1703 (ST-448), and id-1707 (ST452)) were added to the ML tree reconstruction. Sequence alignments were generated in the MUSCLE software [69], and a nucleotide substitution model for each dataset was chosen by means of MEGA 6; statistical branch support was based on 1000 bootstrap iterations. 
To expand the Wolbachia genetic diversity analysis, we created a dataset that included additional MLST profiles. The MLST profiles were chosen according to allele identity toward variants observed in Acrididae isolates ('one allele criterion', see details in refs. [46,62]. Briefly, we took an ST from the Public Databases for Molecular Typing and Microbial Genome Diversity (PubMLST) [70] if it contained the same allele as any of the loci found in our study. Because the number of alleles in our study was unique, we also included STs with the most closely related alleles. To present the phylogenetic relationships of profiles in this dataset, we reconstructed an unrooted phylogenetic network in SplitsTree4 [71] using the neighbour-net method [72]. In addition, we conducted a comprehensive phylogenetic analysis of the data on Wolbachia diversity discovered in Ch. parallelus [44] and in the above-mentioned dataset. We retrieved alleles from GenBank, assembled concatenated sequences, aligned the sequences, excluded redundant parts, and reconstructed the unrooted phylogenetic network.

\section{Conclusions}

Many Acrididae species harbour Wolbachia symbionts. Nonetheless, Wolbachia genetic diversity is rather low among these hosts: (i) there are strains of only supergroups $\mathrm{B}$ and F, (ii) genetic variation is narrow within each supergroup. These data indicate mas-sive Wolbachia horizontal transmission among Acrididae hosts. Specific content of Wolbachia alleles in Acrididae hosts can be used for identifying route(s) and mechanism(s) of Wolbachia horizontal transmission.

Supplementary Materials: The following supporting information can be downloaded at: https:// www.mdpi.com/article/10.3390/ijms23020853/s1.

Author Contributions: Conceptualization, Y.I.; Data curation, Y.I., A.B.; Formal analysis, Y.I.; Investigation, Y.I., M.D. and R.B.; Methodology, Y.I., M.D. and A.B.; Supervision, Y.I. and A.B.; Writingoriginal draft, Y.I.; Writing —-review \& editing, Y.I. All authors have read and agreed to the published version of the manuscript.

Funding: This work was supported by Russian Foundation for Basic Research grant No. 19-0400983, by Ministry of Science and Higher Education (Russian Federation) grant No. 121031800061-7 (to Y.I., M.D. and R.B.) and by the Federal Fundamental Scientific Research Programme grant No. FWSG-2021-0004 (to A.B.).

Institutional Review Board Statement: Not applicable.

Informed Consent Statement: Not applicable.

Data Availability Statement: Not applicable.

Acknowledgments: We are grateful to Andrey Broshkov for technical support and to Nikolai Shevchuk and two anonymous reviewers for valuable comments and suggestions.

Conflicts of Interest: The authors declare no conflict of interest.

\section{References}

1. Gerth, M.; Gansauge, M.-T.; Weigert, A.; Bleidorn, C. Phylogenomic analyses uncover origin and spread of the Wolbachia pandemic. Nat. Commun. 2014, 5, 5117. [CrossRef]

2. Werren, J.H. Biology of Wolbachia. Annu. Rev. Entomol. 1997, 42, 587-609. [CrossRef] [PubMed]

3. Zug, R.; Hammerstein, P. Bad guys turned nice? A critical assessment of Wolbachia mutualisms in arthropod hosts. Biol. Rev. 2015, 90, 89-111. [CrossRef]

4. Werren, J.H.; Windsor, D.; Guo, L.R. Distribution of Wolbachia among neotropical arthropods. Proc. R. Soc. Lond. Ser. B Biol. Sci. 1995, 262, 197-204. [CrossRef]

5. Glowska, E.; Dragun-Damian, A.; Dabert, M.; Gerth, M. New Wolbachia supergroups detected in quill mites (Acari: Syringophilidae). Infect. Genet. Evol. 2015, 30, 140-146. [CrossRef]

6. Laidoudi, Y.; Levasseur, A.; Medkour, H.; Maaloum, M.; Ben Khedher, M.; Sambou, M.; Bassene, H.; Davoust, B.; Fenollar, F.; Raoult, D.; et al. An Earliest Endosymbiont, Wolbachia massiliensis sp. nov., Strain PL13 from the Bed Bug (Cimex hemipterus), Type Strain of a New Supergroup T. Int. J. Mol. Sci. 2020, 21, 8064. [CrossRef] [PubMed] 
7. Ferri, E.; Bain, O.; Barbuto, M.; Martin, C.; Lo, N.; Uni, S.; Landmann, F.; Baccei, S.G.; Guerrero, R.; Lima, S.D.S.; et al. New Insights into the Evolution of Wolbachia Infections in Filarial Nematodes Inferred from a Large Range of Screened Species. PLoS ONE 2011, 6, e20843. [CrossRef]

8. Campbell, B.C.; Bragg, T.S.; Turner, C.E. Phylogeny of symbiotic bacteria of four weevil species (coleoptera: Curculionidae) based on analysis of $16 \mathrm{~S}$ ribosomal DNA. Insect Biochem. Mol. Biol. 1992, 22, 415-421. [CrossRef]

9. Lo, N.; Casiraghi, M.; Salati, E.; Bazzocchi, C.; Bandi, C. How Many Wolbachia Supergroups Exist? Mol. Biol. Evol. 2002, 19, 341-346. [CrossRef]

10. Sontowski, R.; Bernhard, D.; Bleidorn, C.; Schlegel, M.; Gerth, M. Wolbachia distribution in selected beetle taxa characterized by PCR screens and MLST data. Ecol. Evol. 2015, 5, 4345-4353. [CrossRef]

11. Liu, Y.; He, B.; Li, F.; Li, K.; Zhang, L.; Li, X.; Zhao, L. Molecular Identification of Bartonella melophagi and Wolbachia Supergroup F from Sheep Keds in Xinjiang, China. Korean J. Parasitol. 2018, 56, 365-370. [CrossRef]

12. Baldo, L.; Hotopp, J.D.; Jolley, K.A.; Bordenstein, S.R.; Biber, S.A.; Choudhury, R.R.; Hayashi, C.; Maiden, M.C.J.; Tettelin, H.; Werren, J.H. Multilocus Sequence Typing System for the Endosymbiont Wolbachia pipientis. Appl. Environ. Microbiol. 2006, 72 , 7098-7110. [CrossRef] [PubMed]

13. Jäckel, R.; Mora, D.; Dobler, S. Evidence for selective sweeps byWolbachiainfections: Phylogeny ofAlticaleaf beetles and their reproductive parasites. Mol. Ecol. 2013, 22, 4241-4255. [CrossRef] [PubMed]

14. Ramalho, M.O.; Moreau, C.S. The Evolution and Biogeography of Wolbachia in Ants (Hymenoptera: Formicidae). Diversity 2020, 12, 426. [CrossRef]

15. Zimmermann, B.L.; Cardoso, G.M.; Bouchon, D.; Pezzi, P.H.; Palaoro, A.V.; Araujo, P.B. Supergroup F Wolbachia in terrestrial isopods: Horizontal transmission from termites? Evol. Ecol. 2021, 35, 165-182. [CrossRef]

16. Lorenzo-Carballa, M.O.; Torres-Cambas, Y.; Heaton, K.; Hurst, G.D.D.; Charlat, S.; Sherratt, T.N.; Van Gossum, H.; Cordero-Rivera, A.; Beatty, C.D. Widespread Wolbachia infection in an insular radiation of damselflies (Odonata, Coenagrionidae). Sci. Rep. 2019, 9, 11933. [CrossRef]

17. Baldo, L.; Prendini, L.; Corthals, A.; Werren, J.H. Wolbachia Are Present in Southern African Scorpions and Cluster with Supergroup F. Curr. Microbiol. 2007, 55, 367-373. [CrossRef]

18. Niehuis, O.; Hartig, G.; Grath, S.; Pohl, H.; Lehmann, J.; Tafer, H.; Donath, A.; Krauss, V.; Eisenhardt, C.; Hertel, J.; et al. Genomic and Morphological Evidence Converge to Resolve the Enigma of Strepsiptera. Curr. Biol. 2012, 22, 1309-1313. [CrossRef]

19. Casiraghi, M.; Bordenstein, S.R.; Baldo, L.; Lo, N.; Beninati, T.; Wernegreen, J.J.; Werren, J.; Bandi, C. Phylogeny of Wolbachia pipientis based on gltA, groEL and ftsZ gene sequences: Clustering of arthropod and nematode symbionts in the F supergroup, and evidence for further diversity in the Wolbachia tree. Microbiology 2005, 151, 4015-4022. [CrossRef] [PubMed]

20. Lo, N.; Evans, T.A. Phylogenetic diversity of the intracellular symbiont Wolbachia in termites. Mol. Phylogenet. Evol. 2007, 44, 461-466. [CrossRef]

21. Hellemans, S.; Kaczmarek, N.; Marynowska, M.; Calusinska, M.; Roisin, Y.; Fournier, D. Bacteriome-associated Wolbachia of the parthenogenetic termite Cavitermes tuberosus. FEMS Microbiol. Ecol. 2019, 95, fiy235. [CrossRef]

22. Vavre, F.; Fleury, F.; Lepetit, D.; Fouillet, P.; Bouletreau, M. Phylogenetic evidence for horizontal transmission of Wolbachia in host-parasitoid associations. Mol. Biol. Evol. 1999, 16, 1711-1723. [CrossRef] [PubMed]

23. Kittayapong, P.; Jamnongluk, W.; Thipaksorn, A.; Milne, J.R.; Sindhusake, C. Wolbachia infection complexity among insects in the tropical rice-field community. Mol. Ecol. 2003, 12, 1049-1060. [CrossRef]

24. Haine, E.R.; Pickup, N.J.; Cook, J.M. Horizontal transmission of Wolbachia in a Drosophila community. Ecol. Entomol. 2005, 30, 464-472. [CrossRef]

25. Sintupachee, S.; Milne, J.R.; Poonchaisri, S.; Baimai, V.; Kittayapong, P. Closely related Wolbachia strains within the pumpkin arthropod community and the potential for horizontal transmission via the plant. Microb. Ecol. 2006, 51, 294-301. [CrossRef]

26. Le Clec'H, W.; Chevalier, F.; Genty, L.; Bertaux, J.; Bouchon, D.; Sicard, M. Cannibalism and Predation as Paths for Horizontal Passage of Wolbachia between Terrestrial Isopods. PLoS ONE 2013, 8, e60232. [CrossRef]

27. Yang, X.-H.; Zhu, D.-H.; Liu, Z.; Zhao, L.; Su, C.-Y. High Levels of Multiple Infections, Recombination and Horizontal Transmission of Wolbachia in the Andricus mukaigawae (Hymenoptera; Cynipidae) Communities. PLoS ONE 2013, 8, e78970. [CrossRef]

28. Ahmed, M.Z.; Li, S.-J.; Xue, X.; Yin, X.-J.; Ren, S.-X.; Jiggins, F.M.; Greeff, J.; Qiu, B.-L. The Intracellular Bacterium Wolbachia Uses Parasitoid Wasps as Phoretic Vectors for Efficient Horizontal Transmission. PLoS Pathog. 2015, 11, e1004672. [CrossRef]

29. Li, S.J.; Muhammad, Z.A.; Lv, N.; Shi, P.Q.; Wang, X.M.; Huang, J.L.; Qiu, B.L. Plant-mediated horizontal transmission of Wolbachia between whiteflies. Int. Soc. Microb. Ecol. J. 2017, 11, 1019-1028.

30. Johannesen, J. Tracing the history and ecological context of Wolbachia double infection in a specialist host (Urophora cardui)parasitoid (Eurytoma serratulae) system. Ecol. Evol. 2017, 7, 986-996. [CrossRef]

31. Hou, H.Q.; Zhao, G.Z.; Su, C.Y.; Zhu, D.H. Wolbachia prevalence patterns: Horizontal transmission, recombination, and multiple infections in chestnut gall wasp-parasitoid communities. Entomol. Exp. Appl. 2020, 168, 752-765. [CrossRef]

32. Martínez-Rodríguez, P.; Bella, J.L.; Nichols, R.A. Wolbachia Infection in Podisma Pedestris Hybrid Zone. Conference SPECIATION2013, Poster. 2013. Available online: https://www.researchgate.net/publication/236591475_Wolbachia_infection_in_ Podisma_pedestris (accessed on 12 December 2021).

33. Bugrov, A.; Ilinsky, Y.Y.; Strunov, A.; Zhukova, M.; Kiseleva, E.; Akimoto, S.; Tatsuta, H. First evidence of Wolbachia infection in populations of grasshopper Podisma sapporensis (Orthoptera: Acrididae). Èntomol. Sci. 2016, 19, 296-300. [CrossRef] 
34. Werren, J.H.; Windsor, D.M. Wolbachia infection frequencies in insects: Evidence of a global equilibrium? Proc. R. Soc. Lond. Ser. B Biol. Sci. 2000, 267, 1277-1285. [CrossRef]

35. Weeks, A.R.; Velten, R.; Stouthamer, R. Incidence of a new sex-ratio-distorting endosymbiotic bacterium among arthropods. Proc. R. Soc. Lond. Ser. B Biol. Sci. 2003, 270, 1857-1865. [CrossRef]

36. Duron, O.; Bouchon, D.; Boutin, S.; Bellamy, L.; Zhou, L.; Engelstädter, J.; Hurst, G.D. The diversity of reproductive parasites among arthropods: Wolbachia do not walk alone. BMC Biol. 2008, 6, 27. [CrossRef] [PubMed]

37. Kumalawati, D.A.; Supriyati, E.; Rachman, M.P.; Oktriani, R.; Kurniasari, I.; Candrasari, D.S.; Hidayati, L.; Handayaningsih, A.E.; Probowati, V.C.; Arianto, B. Wolbachia infection prevalence as common insects' endosymbiont in the rural area of Yogyakarta, Indonesia. Biodivers. J. Biol. Divers. 2020, 21, 5608-5614. [CrossRef]

38. Dillon, R.; Webster, G.; Weightman, A.; Dillon, V.; Blanford, S.; Charnley, A. Composition of Acridid gut bacterial communities as revealed by $16 \mathrm{~S}$ rRNA gene analysis. J. Invertebr. Pathol. 2008, 97, 265-272. [CrossRef]

39. Martinez, P.; Del Castillo, P.; Bella, J. Cytological detection of Wolbachia in squashed and paraffin embedded insect tissues. Biotech Histochem. 2010, 84, 347-353. [CrossRef]

40. Zabal-Aguirre, M.; Arroyo, F.; Bella, J.L. Distribution of Wolbachia infection in Chorthippus parallelus populations within and beyond a Pyrenean hybrid zone. Heredity 2010, 104, 174-184. [CrossRef]

41. Sarasa, J.; Bernal, A.; Fernandez-Calvin, B.; Bella, J. WolbachiaInduced Cytogenetical Effects as Evidenced in Chorthippus parallelus (Orthoptera). Cytogenet. Genome Res. 2013, 139, 36-43. [CrossRef]

42. Funkhouser-Jones, L.J.; Sehnert, S.R.; Martínez-Rodríguez, P.; Toribio-Fernández, R.; Pita, M.; Bella, J.L.; Bordenstein, S.R. Wolbachia co-infection in a hybrid zone: Discovery of horizontal gene transfers from two Wolbachia supergroups into an animal genome. PeerJ 2015, 3, e1479. [CrossRef]

43. Toribio-Fernández, R.; Bella, J.L.; Martínez-Rodríguez, P.; Funkhouser-Jones, L.J.; Bordenstein, S.R.; Pita, M. Chromosomal localization of Wolbachia inserts in the genomes of two subspecies of Chorthippus parallelus forming a Pyrenean hybrid zone. Chromosom. Res. 2017, 25, 215-225. [CrossRef]

44. Martínez-Rodríguez, P.; Bella, J.L. Chorthippus parallelus and Wolbachia: Overlapping Orthopteroid and Bacterial Hybrid Zones Front. Genet. 2018, 9, 604. [CrossRef] [PubMed]

45. Martínez-Rodríguez, P.; Rolán-Alvarez, E.; del Mar Pérez-Ruiz, M.; Arroyo-Yebras, F.; Carpena-Catoira, C.; Carvajal-Rodríguez, A.; Bella, J.L. Geographic and Temporal Variation of Distinct Intracellular Endosymbiont Strains of Wolbachia sp. in the Grasshopper Chorthippus parallelus: A Frequency-Dependent Mechanism? Microb. Ecol. 2019, 77, 1036-1047. [CrossRef]

46. Ilinsky, Y.; Kosterin, O.E. Molecular diversity of Wolbachia in Lepidoptera: Prevalent allelic content and high recombination of MLST genes. Mol. Phylogenet. Evol. 2017, 109, 164-179. [CrossRef]

47. Tseng, S.-P.; Hsu, P.-W.; Lee, C.-C.; Wetterer, J.K.; Hugel, S.; Wu, L.-H.; Lee, C.-Y.; Yoshimura, T.; Yang, C.-C.S. Evidence for Common Horizontal Transmission of Wolbachia among Ants and Ant Crickets: Kleptoparasitism Added to the List. Microorganisms 2020, 8, 805. [CrossRef] [PubMed]

48. Klasson, L.; Kumar, N.; Bromley, R.; Sieber, K.; Flowers, M.; Ott, S.H.; Tallon, L.J.; Andersson, S.G.E.; Hotopp, J.C.D. Extensive duplication of the Wolbachia DNA in chromosome four of Drosophila ananassae. BMC Genom. 2014, 15, 1097. [CrossRef]

49. Koutsovoulos, G.; Makepeace, B.; Tanya, V.N.; Blaxter, M. Palaeosymbiosis Revealed by Genomic Fossils of Wolbachia in a Strongyloidean Nematode. PLoS Genet. 2014, 10, e1004397. [CrossRef]

50. Brelsfoard, C.; Tsiamis, G.; Falchetto, M.; Gomulski, L.M.; Telleria, E.; Alam, U.; Doudoumis, V.; Scolari, F.; Benoit, J.B.; Swain, M.; et al. Presence of Extensive Wolbachia Symbiont Insertions Discovered in the Genome of Its Host Glossina morsitans morsitans. PLoS Negl. Trop. Dis. 2014, 8, e2728. [CrossRef]

51. Conner, W.R.; Blaxter, M.L.; Anfora, G.; Ometto, L.; Rota-Stabelli, O.; Turelli, M. Genome comparisons indicate recent transfer of wR i-like Wolbachia between sister species Drosophila suzukii and D. subpulchrella. Ecol. Evol. 2017, 7, 9391-9404. [CrossRef]

52. Cordaux, R.; Gilbert, C. Evolutionary Significance of Wolbachia-to-Animal Horizontal Gene Transfer: Female Sex Determination and the $f$ Element in the Isopod Armadillidium vulgare. Genes 2017, 8, 186. [CrossRef] [PubMed]

53. Rosselló-Mora, R.; Amann, R. The species concept for prokaryotes. FEMS Microbiol. Rev. 2001, 25, 39-67. [CrossRef]

54. Cohan, F.M. Bacterial species and speciation. Syst. Biol. 2001, 50, 513-524. [CrossRef] [PubMed]

55. Wiedenbeck, J.; Cohan, F.M. Origins of bacterial diversity through horizontal genetic transfer and adaptation to new ecological niches. FEMS Microbial. Rev. 2011, 35, 957-976. [CrossRef]

56. Pfarr, K.; Foster, J.; Slatko, B.; Hoerauf, A.; Eisen, J.A. On the taxonomic status of the intracellular bacterium Wolbachia pipientis: Should this species name include the intracellular bacteria of filarial nematodes? Int. J. Syst. Evol. Microbiol. 2007, 57 Pt 8, 1677. [CrossRef]

57. Ellegaard, K.M.; Klasson, L.; Näslund, K.; Bourtzis, K.; Andersson, S.G. Comparative genomics of Wolbachia and the bacterial species concept. PLoS Genet. 2013, 9, e1003381. [CrossRef]

58. Ramírez-Puebla, S.T.; Servín-Garcidueñas, L.E.; Ormeño-Orrillo, E.; de León, A.V.P.; Rosenblueth, M.; Delaye, L.; Martínez, J.; Martínez-Romero, E. Species in Wolbachia? Proposal for the designation of 'Candidatus Wolbachia bourtzisii','Candidatus Wolbachia onchocercicola','Candidatus Wolbachia blaxteri','Candidatus Wolbachia brugii','Candidatus Wolbachia taylori','Candidatus Wolbachia collembolicola'and 'Candidatus Wolbachia multihospitum' for the different species within Wolbachia supergroups. Syst. Appl. Microbiol. 2015, 38, 390-399. 
59. Lindsey, A.R.; Bordenstein, S.R.; Newton, I.L.; Rasgon, J.L. Wolbachia pipientis should not be split into multiple species: A response to Ramírez-Puebla et al., "Species in Wolbachia? Proposal for the designation of 'Candidatus Wolbachia bourtzisii','Candidatus Wolbachia onchocercicola','Candidatus Wolbachia blaxteri','Candidatus Wolbachia brugii','Candidatus Wolbachia taylori','Candidatus Wolbachia collembolicola'and 'Candidatus Wolbachia multihospitum'for the different species within Wolbachia supergroups”. Syst. Appl. Microbiol. 2016, 39, 220-222.

60. Baldo, L.; Bordenstein, S.; Wernegreen, J.J.; Werren, J.H. Widespread recombination throughout Wolbachia genomes. Mol. Biol. Evol. 2006, 23, 437-449. [CrossRef]

61. Baldo, L.; Werren, J.H. Revisiting Wolbachia supergroup typing based on WSP: Spurious lineages and discordance with MLST. Curr. Microbiol. 2007, 55, 81-87. [CrossRef]

62. Shaikevich, E.; Bogacheva, A.; Rakova, V.; Ganushkina, L.; Ilinsky, Y. Wolbachia symbionts in mosquitoes: Intra-and intersupergroup recombinations, horizontal transmission and evolution. Mol. Phylogenetics Evol. 2019, 134, 24-34. [CrossRef] [PubMed]

63. Wang, X.; Xiong, X.; Cao, W.; Zhang, C.; Werren, J.H.; Wang, X. Phylogenomic Analysis of Wolbachia Strains Reveals Patterns of Genome Evolution and Recombination. Genome Biol. Evol. 2020, 12, 2508-2520. [CrossRef]

64. Scholz, M.; Albanese, D.; Tuohy, K.; Donati, C.; Segata, N.; Rota-Stabelli, O. Large scale genome reconstructions illuminate Wolbachia evolution. Nat. Commun. 2020, 11, 5235. [CrossRef]

65. Badawi, M.; Giraud, I.; Vavre, F.; Grève, P.; Cordaux, R. Signs of Neutralization in a Redundant Gene Involved in Homologous Recombination in Wolbachia Endosymbionts. Genome Biol. Evol. 2014, 6, 2654-2664. [CrossRef]

66. Nice, C.C.; Gompert, Z.; Forister, M.L.; Fordyce, J.A. An unseen foe in arthropod conservation efforts: The case of Wolbachia infections in the Karner blue butterfly. Biol. Conserv. 2009, 142, 3137-3146. [CrossRef]

67. Folmer, O.; Black, M.; Hoeh, W.; Lutz, R.; Vrijenhoek, R. DNA primers for amplification of mitochondrial cytochrome c oxidase subunit I from diverse metazoan invertebrates. Mol. Mar. Biol. Biotechnol. 1994, 3, 294-299. [PubMed]

68. Bykov, R.; Kerchev, I.; Demenkova, M.; Ryabinin, A.; Ilinsky, Y. Sex-Specific Wolbachia Infection Patterns in Populations of Polygraphus proximus Blandford (Coleoptera; Curculionidae: Scolytinae). Insects 2020, 11, 547. [CrossRef] [PubMed]

69. Edgar, R.C. MUSCLE: A multiple sequence alignment method with reduced time and space complexity. BMC Bioinform. 2004, 5, 113. [CrossRef] [PubMed]

70. Jolley, K.A.; Bray, J.E.; Maiden, M.C.J. Open-access bacterial population genomics: BIGSdb software, the PubMLST.org website and their applications. Wellcome Open Res. 2018, 3, 124. [CrossRef]

71. Huson, D.H.; Bryant, D. Application of Phylogenetic Networks in Evolutionary Studies. Mol. Biol. Evol. 2006, 23, 254-267. [CrossRef]

72. Bryant, D.; Moulton, V. Neighbor-net: An Agglomerative Method for the Construction of Planar Phylogenetic Networks. Mol. Biol. Evol. 2004, 21, 255-265. [CrossRef] [PubMed] 